\title{
THE UNIVERSALITY OF THE REZK NERVE
}

\author{
AARON MAZEL-GEE
}

\begin{abstract}
We functorially associate to each relative $\infty$-category $(\mathcal{R}, \mathbf{W})$ a simplicial space $\mathrm{N}_{\infty}^{\mathrm{R}}(\mathcal{R}, \mathbf{W})$, called its Rezk nerve (a straightforward generalization of Rezk's "classification diagram" construction for relative categories). We prove the following local and global universal properties of this construction: (i) that the complete Segal space generated by the Rezk nerve $\mathrm{N}_{\infty}^{\mathrm{R}}(\mathcal{R}, \mathbf{W})$ is precisely the one corresponding to the localization $\mathcal{R} \llbracket \mathbf{W}^{-1} \rrbracket$; and (ii) that the Rezk nerve functor defines an equivalence $\mathcal{R e l C a t}_{\infty} \llbracket \mathbf{W}_{\mathrm{BK}}^{-1} \rrbracket \stackrel{\sim}{\longrightarrow} \operatorname{Cat}_{\infty}$ from a localization of the $\infty$-category of relative $\infty$-categories to the $\infty$-category of $\infty$-categories.
\end{abstract}

\section{Contents}

0. Introduction 1

1. Relative $\infty$-categories and their localizations 4

2. Complete Segal spaces 10

3. The Rezk nerve 12

4. The proof of Theorem $3.8 \quad 18$

References 26

\section{INTRODUCTION}

0.1 . The Rezk nerve. A relative $\infty$-category is a pair $(\mathcal{R}, \mathbf{W})$ of an $\infty$-category $\mathcal{R}$ and a subcategory $\mathbf{W} \subset \mathcal{R}$ containing all the equivalences, called the subcategory of weak equivalences. Freely inverting the weak equivalences, we obtain the localization of this relative $\infty$-category, namely the initial functor

$$
\mathcal{R} \rightarrow \mathcal{R} \llbracket \mathbf{W}^{-1} \rrbracket
$$

from $\mathcal{R}$ which sends all maps in $\mathbf{W}$ to equivalences. In general, it is extremely difficult to access the localization. ${ }^{1}$ To ameliorate this state of affairs, in this paper we provide a novel method of accessing this localization via Rezk's theory of complete Segal spaces.

To describe this, let us first recall that the $\infty$-category $\mathcal{C S S}$ of complete Segal spaces participates in a diagram

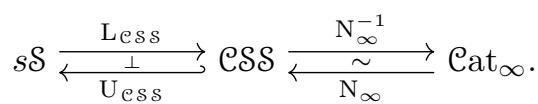

That is, it sits as a reflective subcategory of the $\infty$-category $s \mathcal{S}$ of simplicial spaces, and it is equivalent to the $\infty$-category $\mathrm{Cat}_{\infty}$ of $\infty$-categories. In particular, one can contemplate the complete Segal space (or equivalently, the $\infty$-category) generated by an arbitrary simplicial space $Y$, much as one can contemplate the 1-category generated by an arbitrary simplicial set: this is encoded by the unit

$$
Y \stackrel{\eta}{\rightarrow} \mathrm{L}_{\mathcal{C S S}}(Y)
$$

of the adjunction (where we omit the inclusion functor $U_{\mathcal{E S S}}$ for brevity).

Now, given a relative $\infty$-category $(\mathcal{R}, \mathbf{W})$, its $\boldsymbol{R e z k}$ nerve is a certain simplicial space

$$
\mathrm{N}_{\infty}^{\mathrm{R}}(\mathcal{R}, \mathbf{W}) \in s \mathcal{S}
$$

\footnotetext{
Date: July $12,2018$.

${ }^{1}$ For instance, even in the case that $\mathcal{R}$ is a one-object 1-category and we are only interested in its 1 -categorical localization, i.e. the composite $\mathcal{R} \rightarrow \mathcal{R} \llbracket \mathbf{W}^{-1} \rrbracket \rightarrow h o\left(\mathcal{R} \llbracket \mathbf{W}^{-1} \rrbracket\right) \simeq \mathcal{R}\left[\mathbf{W}^{-1}\right]$ - that is, in the case that we are interested in freely inverting certain elements of a monoid -, obtaining a concrete description is nevertheless an intractable (in fact, computationally undecidable) task, closely related to the so-called "word problem" for generators and relations in abstract algebra.
} 
which "wants to be" the complete Segal space

$$
\mathrm{N}_{\infty}\left(\mathcal{R} \llbracket \mathbf{W}^{-1} \rrbracket\right) \in \mathcal{C S S}
$$

corresponding to its localization:

- it admits canonical maps

$$
\mathrm{N}_{\infty}(\mathcal{R}) \rightarrow \mathrm{N}_{\infty}^{\mathrm{R}}(\mathcal{R}, \mathbf{W}) \rightarrow \mathrm{N}_{\infty}\left(\mathcal{R} \llbracket \mathbf{W}^{-1} \rrbracket\right),
$$

and moreover

- its construction manifestly dictates that for any $\infty$-category $\mathcal{C}$, the restriction map

$$
\operatorname{hom}_{s \mathcal{S}}\left(\mathrm{N}_{\infty}^{\mathrm{R}}(\mathcal{R}, \mathbf{W}), \mathrm{N}_{\infty}(\mathcal{C})\right) \rightarrow \operatorname{hom}_{s \mathcal{S}}\left(\mathrm{N}_{\infty}(\mathcal{R}), \mathrm{N}_{\infty}(\mathcal{C})\right) \simeq \operatorname{hom}_{\mathcal{C a t}_{\infty}}(\mathcal{R}, \mathcal{C})
$$

factors through the subspace of those functors $\mathcal{R} \rightarrow \mathcal{C}$ sending all maps in $\mathbf{W} \subset \mathcal{R}$ to equivalences in e.

Unfortunately, life is not quite so simple: the Rezk nerve is not generally a complete Segal space (or even a Segal space). ${ }^{2}$ Nevertheless, the second-best-possible thing is true.

Theorem (3.8). The above maps extend to a commutative diagram

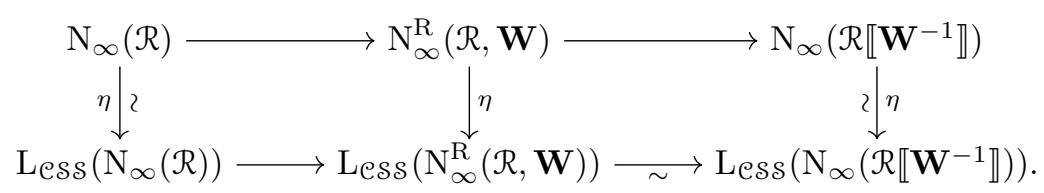

In other words, the complete Segal space generated by the Rezk nerve of $(\mathcal{R}, \mathbf{W})$ is precisely the one corresponding to its localization.

This theorem provides a local universal property of the Rezk nerve: it asserts that the composite

$$
\text { RelCat }_{\infty} \stackrel{\mathrm{N}_{\infty}^{\mathrm{R}}}{\longrightarrow} s \mathcal{\text { Less }} \longrightarrow \text { CSS } \stackrel{\mathrm{N}_{\infty}^{-1}}{\sim} \text { Cat }_{\infty}
$$

takes each relative $\infty$-category $(\mathcal{R}, \mathbf{W})$ to its localization $\mathcal{R} \llbracket \mathbf{W}^{-1} \rrbracket$. However, it says nothing about the effect of this composite on morphisms of relative $\infty$-categories. To this end, we also prove the following.

Theorem (3.9 and 3.12). The above composite is canonically equivalent to the localization functor

$$
\text { RelCat }_{\infty} \rightarrow \text { Cat }_{\infty} .
$$

In particular, denoting by $\mathbf{W}_{\mathrm{BK}} \subset \mathcal{R e l} \mathrm{Cat}_{\infty}$ the subcategory of maps which it takes to equivalences, the above composite induces an equivalence

$$
\operatorname{RelCat}_{\infty} \llbracket \mathbf{W}_{\mathrm{BK}}^{-1} \rrbracket \stackrel{\sim}{\rightarrow} \operatorname{Cat}_{\infty} .
$$

In other words, the Rezk nerve functor does indeed functorially compute localizations of relative $\infty$ categories, and moreover the induced "homotopy theory" on the $\infty$-category $\mathcal{R e l} \mathcal{C a t}_{\infty}$ of relative $\infty$-categories - that is, the relative $\infty$-category structure $\left(\mathcal{R e l} \mathcal{C a t}_{\infty}, \mathbf{W}_{\mathrm{BK}}\right)$ that results therefrom - gives a presentation of the $\infty$-category Cat $_{\infty}$ of $\infty$-categories. We therefore deem this result as capturing the global universal property of the Rezk nerve.

Remark 0.1. The Rezk nerve functor is a close cousin of Rezk's "classification diagram" functor of [Rez01, 3.3]; to emphasize the similarity, we denote the latter functor by

$$
\text { Releat } \stackrel{\mathrm{N}^{\mathrm{R}}}{\longrightarrow} s s \text { Set }
$$

and refer to it as the 1-categorical Rezk nerve. In fact, as we explain in Remark 3.2, this is essentially just the restriction of the $\infty$-categorical Rezk nerve functor, in the sense that there is a canonical commutative diagram

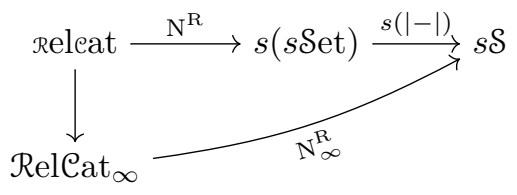

\footnotetext{
${ }^{2}$ We provide sufficient conditions on $(\mathcal{R}, \mathbf{W})$ for its Rezk nerve $\mathrm{N}_{\infty}^{\mathrm{R}}(\mathcal{R}, \mathbf{W})$ to be a (complete) Segal space in [MGd].
} 
in Cat $_{\infty}$. In Remark 3.13, we use this observation to show that our global universal property of the $\infty$ categorical Rezk nerve can be seen as a generalization of work of Barwick-Kan.

0.2. Conventions. Though it stands alone, this paper belongs to a series on model $\infty$-categories. These papers share many key ideas; thus, rather than have the same results appear repeatedly in multiple places, we have chosen to liberally cross-reference between them. To this end, we introduce the following "code names".

\begin{tabular}{||c|c|c||}
\hline \hline title & reference & code \\
\hline \hline Model $\infty$-categories I: some pleasant properties of the $\infty$-category of simplicial spaces & {$[\mathrm{MGa}]$} & $\mathrm{S}$ \\
\hline The universality of the Rezk nerve & $\mathrm{n} / \mathrm{a}$ & $\mathrm{N}$ \\
\hline All about the Grothendieck construction & {$[\mathrm{MGc}]$} & $\mathrm{G}$ \\
\hline Hammocks and fractions in relative $\infty$-categories & {$[\mathrm{MGd}]$} & $\mathrm{H}$ \\
\hline Model $\infty$-categories II: Quillen adjunctions & {$[\mathrm{MGe}]$} & $\mathrm{Q}$ \\
\hline Model $\infty$-categories III: the fundamental theorem & {$[\mathrm{MGf}]$} & $\mathrm{M}$ \\
\hline \hline
\end{tabular}

Thus, for instance, to refer to [MGf, Theorem 1.9], we will simply write Theorem M.1.9. (The letters are meant to be mnemonical: they stand for "simplicial space", "nerve", "Grothendieck", "hammock", "Quillen", and "model", respectively.)

We take quasicategories as our preferred model for $\infty$-categories, and in general we adhere to the notation and terminology of [Lur09a] and [Lur14]. In fact, our references to these two works will be frequent enough that it will be convenient for us to adopt Lurie's convention and use the code names $\mathrm{T}$ and $\mathrm{A}$ for them, respectively.

However, we work invariantly to the greatest possible extent: that is, we primarily work within the $\infty$ category of $\infty$-categories. Thus, for instance, we will omit all technical uses of the word "essential", e.g. we will use the term unique in situations where one might otherwise say "essentially unique" (i.e. parametrized by a contractible space). For a full treatment of this philosophy as well as a complete elaboration of our conventions, we refer the interested reader to $\S$ S.A. The casual reader should feel free to skip this on a first reading; on the other hand, the careful reader may find it useful to peruse that section before reading the present paper. For the reader's convenience, we also provide a complete index of the notation that is used throughout this sequence of papers in $\S$ S.B.

0.3. Outline. We now provide a more detailed outline of the contents of this paper.

- In $\S 1$, we undertake a study of relative $\infty$-categories and their localizations.

- In $\S 2$, we briefly review the theory of complete Segal spaces.

- In $\S 3$, we introduce the Rezk nerve and state its local and global universal properties. We give a proof of the global universal property which relies on the local one, but we defer the proof of the local one to $\S 4$.

- In $\S 4$, we prove the local universal property of the Rezk nerve. Though much of the proof is purely formal, at its heart it ultimately relies on some rather delicate model-categorical arguments.

0.4. Acknowledgments. We heartily thank Zhen Lin Low, Eric Peterson, Chris Schommer-Pries, and Mike Shulman for many (sometimes extremely extended) discussions regarding the material in this paper, particularly the proof of Lemma 4.3. It is also our pleasure to thank Katherine de Kleer for writing a Python script verifying the identities for the simplicial homotopies defined therein. ${ }^{3}$ Lastly, we thank the NSF graduate research fellowship program (grant DGE-1106400) for its financial support during the time that this work was carried out.

\footnotetext{
${ }^{3}$ This script is readily available upon request.
} 


\section{Relative $\infty$-CATEGORIES AND THEIR LOCALIZATIONS}

Given an $\infty$-category and some chosen subset of its morphisms, we are interested in freely inverting those morphisms. In order to codify these initial data, we introduce the following.

Definition 1.1. A relative $\infty$-category is a pair $(\mathcal{R}, \mathbf{W})$ of an $\infty$-category $\mathcal{R}$ and a subcategory $\mathbf{W} \subset \mathcal{R}$, called the subcategory of weak equivalences, such that $\mathbf{W}$ contains all the equivalences (and in particular, all the objects) in $\mathcal{R}$. These form the evident $\infty$-category $\mathcal{R e l}$ at $_{\infty} \cdot{ }^{4}$ Weak equivalences will be denoted by the symbol $\underset{\rightarrow}{\rightarrow}$. Though we will of course write $\mathcal{R}$ for the $\infty$-category obtained by forgetting $\mathbf{W}$, to ease notation we will also sometimes simply write $\mathcal{R}$ for the pair $(\mathcal{R}, \mathbf{W})$. We write $\operatorname{Rel}$ at $\subset \mathcal{R e l} \mathcal{C a t}_{\infty}$ for the full subcategory on those relative $\infty$-categories $(\mathcal{R}, \mathbf{W})$ such that $\mathcal{R} \in \mathcal{C}_{\text {at }} \subset \mathcal{C a t}_{\infty}$.

Remark 1.2. As we are working invariantly, our Definition 1.1 is not quite a generalization of the 1-category Releat of relative categories as given e.g. in [BK12, 3.1] or [LMG15, Definition 3.1], an object of which is a strict category $\mathcal{R} \in$ eat (see subitem $\mathrm{S} . \mathrm{A}(4)(\mathrm{c})$ ) equipped with a wide subcategory $\mathbf{W} \subset \mathcal{R}$ (i.e. one containing all the objects). For emphasis, we will therefore sometimes refer to objects of releat as strict relative categories.

In addition to being the only meaningful variant in the invariant world, Definition 1.1 allows for a clean and aesthetically appealing definition of localization, namely as a left adjoint (see Definition 1.8). In any case, as we are ultimately only interested in relative $\infty$-categories because we are interested in their localizations, this requirement is no real loss.

Despite these differences, there is an evident functor

$$
\text { Releat } \rightarrow \text { RelCat, }
$$

to which we will refer on occasion.

Notation 1.3. In order to disambiguate our notation associated to various relative $\infty$-categories, we introduce the following conventions.

- When multiple relative $\infty$-categories are under discussion, we will sometimes decorate them for clarity. For instance, we may write $\left(\mathcal{R}_{1}, \mathbf{W}_{1}\right)$ and $\left(\mathcal{R}_{2}, \mathbf{W}_{2}\right)$ to denote two arbitrary relative $\infty$ categories, or we may instead write $\left(\mathcal{J}, \mathbf{W}_{\mathcal{J}}\right)$ and $\left(\mathcal{J}, \mathbf{W}_{\mathcal{J}}\right)$.

- Moreover, we will eventually study certain "named" relative $\infty$-categories; for example, there is a Barwick-Kan relative structure on RelCat $_{\infty}$ itself (see Definition 1.16). We will always subscript the subcategory of weak equivalences of such a relative $\infty$-category with (an abbreviation of) its name; for example, we will write $\mathbf{W}_{\mathrm{BK}} \subset \mathcal{R}$ elCat ${ }_{\infty}$. We may also merely similarly subscript the ambient $\infty$-category to denote the relative $\infty$-category; for example, we will write $\left(\mathcal{R e l}_{\mathrm{Cat}_{\infty}}\right)_{\mathrm{BK}}=$ $\left(\right.$ RelCat $\left._{\infty}, \mathbf{W}_{\mathrm{BK}}\right)$.

- Finally, there will occasionally be two different $\infty$-categories with relative structures of the same name. In such cases, if disambiguation is necessary we will additionally superscript the subcategory of weak equivalences with the name of the ambient $\infty$-category. For instance, we would write $\mathbf{W}_{\mathrm{BK}}^{\text {Releat }_{\infty}} \subset \mathcal{R}^{\text {RelCat }}{ }_{\infty}$ to distinguish it from the subcategory $\mathbf{W}_{\mathrm{BK}}^{\text {Releat }} \subset$ Releat.

We have the following fundamental source of examples of relative $\infty$-categories.

Example 1.4. If $\mathcal{R} \rightarrow \mathcal{C}$ is any functor of $\infty$-categories, we can define a relative $\infty$-category $(\mathcal{R}, \mathbf{W})$ by declaring $\mathbf{W} \subset \mathcal{R}$ to be the subcategory on those maps that are sent to equivalences in $\mathcal{C}$. Note that $\mathbf{W} \subset \mathcal{R}$ will automatically have the two-out-of-three property.

Definition 1.5. In the situation of Example 1.4, we will say that the functor $\mathcal{R} \rightarrow \mathcal{C}$ creates the subcategory $\mathbf{W} \subset \mathcal{R}$.

We will make heavy use of the following construction.

\footnotetext{
${ }^{4}$ To be precise, one can view $\operatorname{RelCat}_{\infty} \simeq \operatorname{Fun}^{\text {surj mono }}\left([1]\right.$, Cat $\left._{\infty}\right) \subset \mathrm{Fun}([1]$, Cat $\infty)$ as the full subcategory on those functors selecting the inclusion of a surjective monomorphism.
} 
Notation 1.6. Given any $\left(\mathcal{R}_{1}, \mathbf{W}_{1}\right),\left(\mathcal{R}_{2}, \mathbf{W}_{2}\right) \in \mathcal{R e l C a t}_{\infty}$, we define

$$
\left(\operatorname{Fun}\left(\mathcal{R}_{1}, \mathcal{R}_{2}\right)^{\mathcal{R e l}}, \operatorname{Fun}\left(\mathcal{R}_{1}, \mathcal{R}_{2}\right)^{\mathbf{W}}\right) \in \operatorname{RelCat}_{\infty}
$$

by setting

$$
\operatorname{Fun}\left(\mathcal{R}_{1}, \mathcal{R}_{2}\right)^{\mathcal{R e l}} \subset \operatorname{Fun}\left(\mathcal{R}_{1}, \mathcal{R}_{2}\right)
$$

to be the full subcategory on those functors which send $\mathbf{W}_{1} \subset \mathcal{R}_{1}$ into $\mathbf{W}_{2} \subset \mathcal{R}_{2}$, and setting

$$
\operatorname{Fun}\left(\mathcal{R}_{1}, \mathcal{R}_{2}\right)^{\mathbf{W}} \subset \operatorname{Fun}\left(\mathcal{R}_{1}, \mathcal{R}_{2}\right)^{\mathcal{R e l}}
$$

to be the (generally non-full) subcategory on the natural weak equivalences. ${ }^{5}$ It is not hard to see that this defines an internal hom bifunctor for $\left(\mathcal{R e l C a t}_{\infty}, \times\right)$.

It will be useful to have the following terminology.

Definition 1.7. If $\mathcal{C}$ is any $\infty$-category, we call $(\mathcal{C}, \mathcal{C} \simeq$ ) the associated minimal relative $\infty$-category and we call $(\mathcal{C}, \mathcal{C})$ the associated maximal relative $\infty$-category. These define fully faithful inclusions

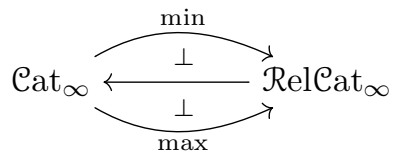

which are respectively left and right adjoint to the forgetful functor $\mathcal{R e l C a t}_{\infty} \stackrel{\mathrm{U}_{\mathcal{R e l}}}{\longrightarrow} \operatorname{Cat}_{\infty}$ sending $(\mathcal{R}, \mathbf{W})$ to $\mathcal{R}$. For $[n] \in \boldsymbol{\Delta} \subset$ Cat $_{\infty}$, we will use the abbreviation $[n]_{\mathbf{W}}=\max ([n])$, since these relative categories will appear quite often; correspondingly, we will also make the implicit identification $[n]=\min ([n])$.

We now come to our central object of interest.

Definition 1.8. The functor min : Cat $_{\infty} \rightarrow$ RelCat $_{\infty}$ also admits a left adjoint

$$
\text { Releat }_{\infty} \stackrel{\mathscr{L}}{\longrightarrow} \text { Cat }_{\infty}
$$

which we refer to as the localization functor on relative $\infty$-categories. For a relative $\infty$-category $(\mathcal{R}, \mathbf{W}) \in$ $\mathcal{R e l C a t}_{\infty}$, we will often write $\mathcal{R} \llbracket \mathbf{W}^{-1} \rrbracket=\mathscr{L}(\mathcal{R}, \mathbf{W})$; we only write $\mathscr{L}$ since the notation $(-) \llbracket(-)^{-1} \rrbracket$ is a bit unwieldy. Explicitly, its value on $(\mathcal{R}, \mathbf{W}) \in \mathcal{R}$ elCat $\infty$ can be obtained as the pushout

$$
\mathcal{R} \llbracket \mathbf{W}^{-1} \rrbracket \simeq \operatorname{colim}(\underbrace{}_{\mathbf{W}^{\text {gpd }}} \underset{\mathcal{R}}{\mathbf{W}})^{\perp}
$$

in $\mathrm{Cat}_{\infty}$ (and the functor itself can be obtained by applying this construction in families).

Remark 1.9. Using model categories, one can of course compute the pushout in Cat Cof $_{\infty}$ Definition 1.8 by

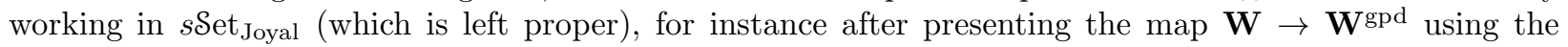
derived unit of the Quillen adjunction id : $s \mathcal{S e t}_{\text {Joyal }} \rightleftarrows s \mathcal{S e t}_{\mathrm{KQ}}$ : id, i.e. after taking a fibrant replacement via a cofibration in $s \mathcal{S e t}_{\mathrm{KQ}}$ of a quasicategory presenting $\mathbf{W}$. However, note that this derived unit can be quite difficult to describe in practice, and moreover the resulting pushout will generally still be very far from being a quasicategory. Equally inexplicitly, one can also obtain a quasicategory presenting $\mathcal{R} \llbracket \mathbf{W}^{-1} \rrbracket$ by computing a fibrant replacement in the marked model structure of Proposition T.3.1.3.7 (i.e. in the specialization of the model structure given there to the case where the base is the terminal object $\mathrm{pt}_{s \mathrm{set}}$ ).

Remark 1.10. We will also use the term "localization" to refer to the canonical map $\mathcal{R} \rightarrow \mathcal{R} \llbracket \mathbf{W}^{-1} \rrbracket$ in $\operatorname{Cat}_{\infty}$ satisfying the universal property that for any $\mathcal{C} \in \mathcal{C} a t_{\infty}$, the restriction

$$
\operatorname{hom}_{\mathcal{C a t}_{\infty}}\left(\mathcal{R} \llbracket \mathbf{W}^{-1} \rrbracket, \mathcal{C}\right) \rightarrow \operatorname{hom}_{\mathcal{C a t}_{\infty}}(\mathcal{R}, \mathcal{C})
$$

defines an equivalence onto the subspace

$$
\operatorname{hom}_{\mathcal{R e l e a t}_{\infty}}((\mathcal{R}, \mathbf{W}), \min (\mathcal{C})) \subset \operatorname{hom}_{\mathcal{C a t}_{\infty}}(\mathcal{R}, \mathcal{C})
$$

\footnotetext{
${ }^{5}$ If we consider $\operatorname{Releat}_{\infty} \subset \operatorname{Fun}([1]$, Cat $\infty)$, then $\operatorname{Fun}\left(\mathcal{R}_{1}, \mathcal{R}_{2}\right)^{\mathcal{R e l}}$ is simply the $\infty$-category of natural transformations.
} 
of those functors which take $\mathbf{W}$ into $\mathcal{C}^{\simeq}{ }^{6}$ Thus, by definition the map $\mathcal{R} \rightarrow \mathcal{R} \llbracket \mathbf{W}^{-1} \rrbracket$ is an epimorphism in $\mathrm{Cat}_{\infty}$.

Example 1.11. The localization of a minimal relative $\infty$-category $\min (\mathcal{C})=(\mathcal{C}, \mathcal{C} \simeq)$ is simply the identity functor $\mathcal{C} \stackrel{\sim}{\rightarrow} \mathcal{C}$.

Example 1.12. The localization of a maximal relative $\infty$-category $\max (\mathcal{C})=(\mathfrak{C}, \mathcal{C})$ is the groupoid completion functor $\mathcal{C} \rightarrow \mathcal{C}^{\text {gpd }}$ (i.e. the component at $\mathcal{C}$ of the unit of the adjunction $(-)^{\text {gpd }}: \mathcal{C a t}_{\infty} \rightleftarrows \mathcal{S}: \mathrm{U}_{\mathcal{S}}$ ).

Example 1.13. Given a left localization adjunction $\mathrm{L}: \mathcal{C} \rightleftarrows \mathrm{LC}: \mathrm{U}$, if we define $\mathbf{W} \subset \mathcal{C}$ to be created by $\mathcal{C} \stackrel{\mathrm{L}}{\rightarrow} \mathrm{LC}$, then the localization of $(\mathcal{C}, \mathbf{W})$ is precisely $\mathcal{C} \stackrel{\mathrm{L}}{\rightarrow} \mathrm{LC}:$ that is, the functor $\mathcal{C} \stackrel{\mathrm{L}}{\rightarrow}$ LC induces an equivalence $\mathcal{C} \llbracket \mathbf{W}^{-1} \rrbracket \stackrel{\sim}{\rightarrow} \mathrm{LC}$, which is in fact inverse to the composite $\mathrm{L} \mathcal{C} \stackrel{\mathrm{U}}{\rightarrow} \mathcal{C} \rightarrow \mathcal{C} \llbracket \mathbf{W}^{-1} \rrbracket$. This follows from Proposition T.5.2.7.12, or alternatively from Lemma 1.24 (see Remark 1.25). Of course, a dual statement holds for right localization adjunctions.

For an arbitrary relative $\infty$-category $(\mathcal{R}, \mathbf{W})$, note that the localization map $\mathcal{R} \rightarrow \mathcal{R} \llbracket \mathbf{W}^{-1} \rrbracket$ might not create the subcategory $\mathbf{W} \subset \mathcal{R}$ : there might be strictly more maps in $\mathcal{R}$ which are sent to equivalences in $\mathcal{R} \llbracket \mathbf{W}^{-1} \rrbracket$. This leads us to the following notion.

Definition 1.14. A relative $\infty$-category $(\mathcal{R}, \mathbf{W})$ is called saturated if the localization map $\mathcal{R} \rightarrow \mathcal{R} \llbracket \mathbf{W}^{-1} \rrbracket$ creates the subcategory $\mathbf{W} \subset \mathcal{R}$.

Remark 1.15. If a relative $\infty$-category $(\mathcal{R}, \mathbf{W}) \in \mathcal{R}$ elCat $\infty$ has its subcategory of weak equivalences $\mathbf{W} \subset \mathcal{R}$ created by any functor $\mathcal{R} \rightarrow \mathcal{C}$, then $(\mathcal{R}, \mathbf{W})$ will automatically be saturated. This is true by definition if $\mathbf{W} \subset \mathcal{R}$ is created by the localization functor $\mathcal{R} \rightarrow \mathcal{R} \llbracket \mathbf{W}^{-1} \rrbracket$. More generally, if it is created by any other functor $\mathcal{R} \rightarrow \mathcal{C}$, then in the canonical factorization

$$
\mathcal{R} \rightarrow \mathcal{R} \llbracket \mathbf{W}^{-1} \rrbracket \rightarrow \mathcal{C},
$$

the second functor will be conservative. Hence, it will be also true that the subcategory $\mathbf{W} \subset \mathcal{R}$ is created by the localization map $\mathcal{R} \rightarrow \mathcal{R} \llbracket \mathbf{W}^{-1} \rrbracket$, which reduces us to the previous special case.

Now, we will be using relative $\infty$-categories as "presentations of $\infty$-categories", namely of their localizations. However, a map of relative $\infty$-categories may induce an equivalence on localizations without itself being an equivalence in $\mathcal{R e l} \mathrm{Cat}_{\infty}$. This leads us to the following notion.

Definition 1.16. We define the subcategory $\mathbf{W}_{\mathrm{BK}} \subset$ RelCat $_{\infty}$ of Barwick-Kan weak equivalences to be created by the localization functor $\mathcal{R e l C a t}_{\infty} \stackrel{\mathscr{L}}{\rightarrow} \operatorname{Cat}_{\infty}$. We denote the resulting relative $\infty$-category by $\left(\text { RelCat }_{\infty}\right)_{\mathrm{BK}}=\left(\mathcal{R e l C a t}_{\infty}, \mathbf{W}_{\mathrm{BK}}\right) \in$ RelCat $_{\infty}$.

The following result then justifies our usage of relative $\infty$-categories as "presentations of $\infty$-categories".

Proposition 1.17. The functors in the left localization adjunction $\mathscr{L}:$ Releat $_{\infty} \rightleftarrows$ Cat $_{\infty}:$ min induce inverse equivalences

in $\mathrm{Cat}_{\infty}$.

$$
\text { RelCat }_{\infty} \llbracket \mathbf{W}_{\mathrm{BK}}^{-1} \rrbracket \simeq \operatorname{Cat}_{\infty}
$$

Proof. This is a special case of Example 1.13.

We have the following strengthening of Remark 1.10.

Proposition 1.18. For any $(\mathcal{R}, \mathbf{W}) \in \mathcal{R e l}^{-} a_{\infty}$ and any $\mathrm{C} \in \mathcal{C a t}_{\infty}$, the restriction

$$
\operatorname{Fun}\left(\mathcal{R} \llbracket \mathbf{W}^{-1} \rrbracket, \mathcal{C}\right) \rightarrow \operatorname{Fun}(\mathcal{R}, \mathcal{C})
$$

along the localization functor $\mathcal{R} \rightarrow \mathcal{R} \llbracket \mathbf{W}^{-1} \rrbracket$ defines an equivalence onto the full subcategory of Fun $(\mathcal{R}, \mathcal{C})$ spanned by those functors which take $\mathbf{W}$ into $\mathrm{e} \simeq$.

\footnotetext{
${ }^{6}$ This map can be obtained either by applying $\operatorname{RelCat}_{\infty} \stackrel{\mathscr{L}}{\longrightarrow}$ Cat $\infty$ to the counit $\min (\mathcal{R}) \rightarrow(\mathcal{R}, \mathbf{W})$ of the adjunction $\min \dashv \mathrm{U}_{\mathcal{R e l}}$, or by applying $\mathcal{R e l} \mathrm{Cat}_{\infty} \stackrel{\mathrm{U}_{\mathcal{R e l}}}{\longrightarrow} \mathcal{C a t}_{\infty}$ to the unit $(\mathcal{R}, \mathbf{W}) \rightarrow \min \left(\mathcal{R} \llbracket \mathbf{W}^{-1} \rrbracket\right)$ of the adjunction $\mathscr{L} \dashv \min$.
} 
Proof. We begin by observing that this functor is a monomorphism in Cat $_{\infty}$ : this is because we have a pullback diagram

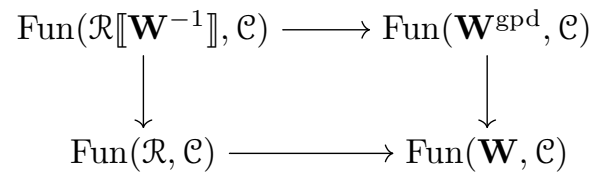

in $\mathrm{Cat}_{\infty}$ in which the right arrow is clearly a monomorphism, and monomorphisms are closed under pullback. So in particular, this functor is the inclusion of a subcategory. Then, to see that it is full, suppose we are given two functors $\mathcal{R} \llbracket \mathbf{W}^{-1} \rrbracket \rightrightarrows \mathcal{C}$, considered as objects of $\operatorname{Fun}\left(\mathcal{R} \llbracket \mathbf{W}^{-1} \rrbracket, \mathcal{C}\right)$. A natural transformation between their images in $\operatorname{Fun}(\mathcal{R}, \mathcal{C})$ is given by a functor $[1] \times \mathcal{R} \rightarrow \mathcal{C}$ which restricts to the the two composites $\mathcal{R} \rightarrow \mathcal{R} \llbracket \mathbf{W}^{-1} \rrbracket \rightrightarrows \mathcal{C}$ on the two objects $0,1 \in[1]$. Since we already know that $\operatorname{Fun}\left(\mathcal{R} \llbracket \mathbf{W}^{-1} \rrbracket, \mathcal{C}\right) \subset$ Fun $(\mathcal{R}, \mathcal{C})$ is the inclusion of a subcategory, it suffices to obtain an extension

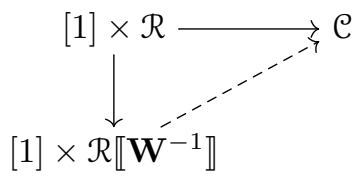

in Cat $_{\infty}$. For this, consider the diagram

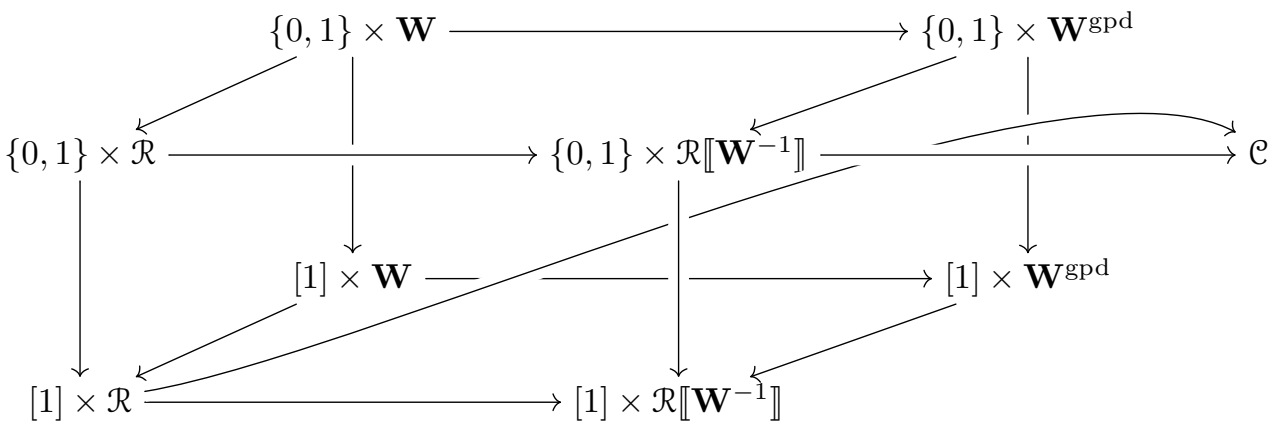

in Cat $_{\infty}$ containing and extending the above data. The bottom square is a pushout since the functor $[1] \times-:$ Cat $_{\infty} \rightarrow$ Cat $_{\infty}$ is a left adjoint, and the back square is a pushout by Lemma 1.20. Together, these observations guarantee the desired extension.

Remark 1.19. Proposition 1.18 implies that Definition 1.8 agrees with Definition A.1.3.4.1.

We now make an easy observation regarding the localization functor, which is necessary for the argument of Proposition 1.18 but will also be useful in its own right.

Lemma 1.20. The localization functor $\mathscr{L}:$ RelCat $_{\infty} \rightarrow$ Cat $_{\infty}$ commutes with finite products.

For the proof of Lemma 1.20, it will be convenient to have the following notion.

Definition 1.21. Let $(\mathcal{C}, \otimes)$ be a closed symmetric monoidal $\infty$-category with internal hom bifunctor

$$
\mathcal{C}^{o p} \times \mathcal{C} \stackrel{\underline{\operatorname{hom}}_{\mathrm{e}}(-,-)}{\longrightarrow} \mathrm{C}
$$

A collection of objects $I$ of $\mathcal{C}$ is called an exponential ideal if we have $\underline{\operatorname{hom}}_{\mathcal{C}}(Y, Z) \in I$ for any $Y \in \mathcal{C}$ and any $Z \in I$. We will use this same terminology to refer to a full subcategory $\mathcal{D} \subset \mathcal{C}$ whose objects form an exponential ideal.

The following straightforward result explains why we are interested in exponential ideals.

Lemma 1.22. Suppose that $(\mathcal{C}, \otimes)$ is a closed symmetric monoidal $\infty$-category, and let $\mathrm{L}: \mathcal{C} \rightleftarrows \mathrm{L} \mathrm{C}: \mathrm{U}$ be a left localization with unit map $\mathrm{id}_{\mathcal{C}} \stackrel{\eta}{\rightarrow} \mathrm{L}$ in $\operatorname{Fun}(\mathcal{C}, \mathcal{C})$ (where we implicitly consider $\left.\mathrm{LC} \subset \mathcal{C}\right) . \quad$ Then, the full subcategory LC $\subset \mathcal{C}$ is an exponential ideal if and only if the natural map $\mathrm{L}(\eta \otimes \eta)$ is an equivalence in $\operatorname{Fun}(\mathcal{C} \times \mathcal{C}, \mathcal{C})$ (i.e. we have

$$
\mathrm{L}(Y \otimes Z) \stackrel{\sim}{\rightarrow} \mathrm{L}(\mathrm{L}(Y) \otimes \mathrm{L}(Z))
$$


in Le for all $Y, Z \in \mathcal{C})$. In particular, if Le is closed under the monoidal structure, then $\mathrm{L} \mathcal{C} \subset \mathcal{C}$ is an exponential ideal if and only if

$$
\mathrm{L}(Y \otimes Z) \simeq \mathrm{L}(Y) \otimes \mathrm{L}(Z)
$$

in Le for all $Y, Z \in \mathcal{C}$.

Proof. Suppose that $\mathrm{L} \mathcal{C} \subset \mathcal{C}$ is an exponential ideal. Then, for any $Y, Z \in \mathcal{C}$ and any test object $W \in \mathrm{LC}$, we have the string of natural equivalences

$$
\begin{aligned}
\operatorname{hom}_{\mathcal{C}}(\mathrm{L}(Y \otimes Z), W) & \simeq \operatorname{hom}_{\mathcal{C}}(Y \otimes Z, W) \simeq \operatorname{hom}_{\mathcal{C}}\left(Y, \underline{\operatorname{hom}}_{\mathcal{C}}(Z, W)\right) \simeq \operatorname{hom}_{\mathcal{C}}\left(\mathrm{L}(Y), \underline{\operatorname{hom}}_{\mathcal{C}}(Z, W)\right) \\
& \simeq \operatorname{hom}_{\mathcal{C}}(\mathrm{L}(Y) \otimes Z, W) \simeq \operatorname{hom}_{\mathcal{C}}(Z \otimes \mathrm{L}(Y), W) \simeq \operatorname{hom} \mathcal{C}\left(Z, \underline{\operatorname{hom}}_{\mathcal{C}}(\mathrm{L}(Y), W)\right) \\
& \simeq \operatorname{hom}_{\mathcal{C}}\left(\mathrm{L}(Z), \underline{\operatorname{hom}}_{\mathfrak{C}}(\mathrm{L}(Y), W)\right) \simeq \operatorname{hom}_{\mathcal{C}}(\mathrm{L}(Z) \otimes \mathrm{L}(Y), W) \\
& \simeq \operatorname{hom}_{\mathcal{C}}(\mathrm{L}(Y) \otimes \mathrm{L}(Z), W) \simeq \operatorname{hom}_{\mathcal{C}}(\mathrm{L}(\mathrm{L}(Y) \otimes \mathrm{L}(Z)), W) .
\end{aligned}
$$

Hence, we have an equivalence $\mathrm{L}(Y \otimes Z) \simeq \mathrm{L}(\mathrm{L}(Y) \otimes \mathrm{L}(Z))$ by the Yoneda lemma applied to the $\infty$-category LC (and it is straightforward to check that this equivalence is indeed induced by the specified map). So $\mathrm{L}(\eta \otimes \eta)$ is an equivalence in $\operatorname{Fun}(\mathcal{C} \times \mathcal{C}, \mathcal{C})$, as desired.

On the other hand, suppose that $\mathrm{L}(Y \otimes Z) \stackrel{\sim}{\rightarrow} \mathrm{L}(\mathrm{L}(Y) \otimes \mathrm{L}(Z))$ for all $Y, Z \in \mathcal{C}$. Then, we have the string of natural equivalences

$$
\begin{aligned}
\operatorname{hom}_{\mathcal{C}}\left(Y, \underline{\operatorname{hom}}_{\mathcal{C}}(Z, W)\right) & \simeq \operatorname{hom}_{\mathcal{C}}(Y \otimes Z, W) \simeq \operatorname{hom}_{\mathcal{C}}(\mathrm{L}(Y \otimes Z), W) \simeq \operatorname{hom}_{\mathcal{C}}(\mathrm{L}(\mathrm{L}(Y) \otimes \mathrm{L}(Z)), W) \\
& \simeq \operatorname{hom}_{\mathcal{C}}(\mathrm{L}(Y) \otimes \mathrm{L}(Z), W) \simeq \operatorname{hom} \mathcal{C}(\mathrm{L}(\mathrm{L}(Y)) \otimes \mathrm{L}(Z), W) \\
& \simeq \operatorname{hom}_{\mathcal{C}}(\mathrm{L}(\mathrm{L}(\mathrm{L}(Y)) \otimes \mathrm{L}(Z)), W) \simeq \operatorname{hom}_{\mathcal{C}}(\mathrm{L}(\mathrm{L}(Y) \otimes Z), W) \\
& \simeq \operatorname{hom}_{\mathcal{C}}(\mathrm{L}(Y) \otimes Z, W) \simeq \operatorname{hom}_{\mathcal{C}}\left(\mathrm{L}(Y), \underline{\operatorname{hom}}_{\mathcal{C}}(Z, W)\right) .
\end{aligned}
$$

Hence, for any map $Y \rightarrow Y^{\prime}$ in $\mathcal{C}$ which localizes to an equivalence $\mathrm{L}(Y) \stackrel{\sim}{\rightarrow} \mathrm{L}\left(Y^{\prime}\right)$ in $\mathrm{LC} \subset \mathcal{C}$, we obtain an equivalence $\operatorname{hom}_{\mathcal{C}}\left(Y, \underline{\operatorname{hom}}_{\mathcal{C}}(Z, W)\right) \stackrel{\operatorname{hom}}{\mathcal{C}}\left(Y^{\prime}, \underline{\operatorname{hom}}_{\mathcal{C}}(Z, W)\right)$. It follows that the object $\underline{\operatorname{hom}}_{\mathcal{C}}(Z, W) \in \mathcal{C}$ is local with respect to the left localization, i.e. that in fact $\underline{\operatorname{hom}}_{\mathcal{C}}(Z, W) \in \mathrm{LC} \subset \mathcal{C}$. So Le $\subset \mathcal{C}$ is an exponential ideal.

With Lemma 1.22 in hand, we now proceed to prove Lemma 1.20.

Proof of Lemma 1.20. The right adjoint min : $\mathrm{Cat}_{\infty} \rightarrow \mathcal{R e l C a t}_{\infty}$ induces an equivalence onto the full subcategory of minimal relative $\infty$-categories. It is easy to see that this is an exponential ideal in $\left(\mathcal{R e l} \mathcal{C a t}_{\infty}, \times\right)$, and so the result follows from Lemma 1.22.

The following useful construction relies on Lemma 1.20.

Remark 1.23. Let $\left(\mathcal{R}_{1}, \mathbf{W}_{1}\right),\left(\mathcal{R}_{2}, \mathbf{W}_{2}\right) \in \mathcal{R e l C a t}_{\infty}$. Then the identity map

$$
\left(\operatorname{Fun}\left(\mathcal{R}_{1}, \mathcal{R}_{2}\right)^{\mathcal{R e l}}, \operatorname{Fun}\left(\mathcal{R}_{1}, \mathcal{R}_{2}\right)^{\mathbf{W}}\right) \rightarrow\left(\operatorname{Fun}\left(\mathcal{R}_{1}, \mathcal{R}_{2}\right)^{\mathcal{R e l}}, \operatorname{Fun}\left(\mathcal{R}_{1}, \mathcal{R}_{2}\right)^{\mathbf{W}}\right)
$$

is adjoint to an evaluation map

$$
\left(\mathcal{R}_{1}, \mathbf{W}_{1}\right) \times\left(\operatorname{Fun}\left(\mathcal{R}_{1}, \mathcal{R}_{2}\right)^{\mathcal{R e l}}, \operatorname{Fun}\left(\mathcal{R}_{1}, \mathcal{R}_{2}\right)^{\mathbf{W}}\right) \rightarrow\left(\mathcal{R}_{2}, \mathbf{W}_{2}\right) .
$$

By Lemma 1.20, applying the localization functor $\mathcal{R e l C a t}_{\infty} \stackrel{\mathscr{L}}{\longrightarrow}$ Cat $_{\infty}$ yields a map

$$
\mathcal{R}_{1} \llbracket \mathbf{W}_{1}^{-1} \rrbracket \times \operatorname{Fun}\left(\mathcal{R}_{1}, \mathcal{R}_{2}\right)^{\mathcal{R e l}} \llbracket\left(\operatorname{Fun}\left(\mathcal{R}_{1}, \mathcal{R}_{2}\right)^{\mathbf{W}}\right)^{-1} \rrbracket \rightarrow \mathcal{R}_{2} \llbracket \mathbf{W}_{2}^{-1} \rrbracket,
$$

which is itself adjoint to a canonical map

$$
\operatorname{Fun}\left(\mathcal{R}_{1}, \mathcal{R}_{2}\right)^{\mathcal{R e l}} \llbracket\left(\operatorname{Fun}\left(\mathcal{R}_{1}, \mathcal{R}_{2}\right)^{\mathbf{W}}\right)^{-1} \rrbracket \rightarrow \operatorname{Fun}\left(\mathcal{R}_{1} \llbracket \mathbf{W}_{1}^{-1} \rrbracket, \mathcal{R}_{2} \llbracket \mathbf{W}_{2}^{-1} \rrbracket\right) .
$$

In particular, precomposing with the localization map for the internal hom-object yields a canonical map

$$
\operatorname{Fun}\left(\mathcal{R}_{1}, \mathcal{R}_{2}\right)^{\mathcal{R e l}} \rightarrow \operatorname{Fun}\left(\mathcal{R}_{1} \llbracket \mathbf{W}_{1}^{-1} \rrbracket, \mathcal{R}_{2} \llbracket \mathbf{W}_{2}^{-1} \rrbracket\right) .
$$

Lemma 1.20 also allows us to prove the following result, which will be useful later and which gives a sense of the interplay between relative $\infty$-categories and their localizations.

Lemma 1.24. Given any $\left(\mathcal{R}_{1} \mathbf{W}_{1}\right),\left(\mathcal{R}_{2}, \mathbf{W}_{2}\right) \in \mathcal{R e l C a t}_{\infty}$ and any pair of maps $\mathcal{R}_{1} \rightrightarrows \mathcal{R}_{2}$ in $\mathcal{R e l C a t}_{\infty}, a$ natural weak equivalence between them induces an equivalence between their induced functors $\mathcal{R}_{1} \llbracket \mathbf{W}_{1}^{-1} \rrbracket \rightrightarrows$ $\mathcal{R}_{2} \llbracket \mathbf{W}_{2}^{-1} \rrbracket$ in Cat $_{\infty}$. 
Proof. A natural weak equivalence corresponds to a map [1] $]_{\mathbf{W}} \times \mathcal{R}_{1} \rightarrow \mathcal{R}_{2}$ in $\mathcal{R e l}$ at ${ }_{\infty}$. By Lemma 1.20 (and Example 1.12), this gives rise to a map [1] $]^{\mathrm{gpd}} \times \mathcal{R}_{1} \llbracket \mathbf{W}_{1}^{-1} \rrbracket \rightarrow \mathcal{R}_{2} \llbracket \mathbf{W}_{2}^{-1} \rrbracket$ in Cat $_{\infty}$, which precisely selects the desired equivalence.

Remark 1.25. Lemma 1.24 allows for a simple proof of Proposition T.5.2.7.12, that a left localization is in particular a free localization. Indeed, given a left localization adjunction $\mathrm{L}: \mathcal{C} \rightleftarrows \mathrm{LC}: \mathrm{U}$, write $\mathbf{W} \subset \mathcal{C}$ for the subcategory created by the functor $\mathrm{L}: \mathcal{C} \rightarrow \mathrm{LC}$. Then, this adjunction gives rise to a pair of maps $(\mathcal{C}, \mathbf{W}) \stackrel{\mathrm{L}}{\rightarrow} \min (\mathrm{LC})$ and $\min (\mathrm{LC}) \stackrel{\mathrm{U}}{\rightarrow}(\mathcal{C}, \mathbf{W})$ in Releat $_{\infty}$. Moreover, the composite

$$
\min (\mathrm{LC}) \stackrel{\mathrm{U}}{\rightarrow}(\mathcal{C}, \mathbf{W}) \stackrel{\mathrm{L}}{\rightarrow} \min (\mathrm{LC})
$$

is an equivalence, while the composite

$$
(\mathcal{C}, \mathbf{W}) \stackrel{\mathrm{L}}{\rightarrow} \min (\mathrm{LC}) \stackrel{\mathrm{U}}{\rightarrow}(\mathcal{C}, \mathbf{W})
$$

is connected to $\mathrm{id}_{(\mathcal{e}, \mathbf{W})}$ by the unit of the natural transformation, which is a componentwise weak equivalence (since for any $Y \in \mathcal{C}$, applying $\mathcal{C} \stackrel{\mathrm{L}}{\rightarrow} \mathrm{L} \mathcal{C}$ to the map $Y \rightarrow \mathrm{L}(Y)$ gives an equivalence $\mathrm{L}(Y) \stackrel{\sim}{\rightarrow} \mathrm{L}(\mathrm{L}(Y))$ ). Hence, it follows that these functors induce inverse equivalences $\mathfrak{C} \llbracket \mathbf{W}^{-1} \rrbracket \simeq$ LC. (From here, one can obtain the actual statement of Proposition T.5.2.7.12 by appealing to Proposition 1.18.)

Lemma 1.24 also has the following special case which will be useful to us.

Lemma 1.26. Given any $\mathcal{C}, \mathcal{D} \in \mathrm{Cat}_{\infty}$ and any pair of maps $\mathcal{C} \rightrightarrows \mathcal{D}$, a natural transformation between

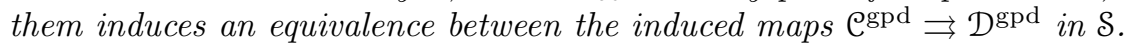

Proof. In light of Example 1.12, this follows from applying Lemma 1.24 in the special case that $\left(\mathcal{R}_{1}, \mathbf{W}_{1}\right)=$ $\max (\mathcal{C})$ and $\left(\mathcal{R}_{2}, \mathbf{W}_{2}\right)=\max (\mathcal{D})$.

Remark 1.27. Lemma 1.26 can also be seen as following from applying Lemma 1.22 to the left localization $(-)^{\text {gpd }}:$ Cat $_{\infty} \rightleftarrows \mathcal{S}: \mathrm{U}_{\mathcal{S}}$. Namely, since the full subcategory $\mathcal{S} \subset$ Cat $_{\infty}$ is an exponential ideal for $\left(\right.$ Cat $\left._{\infty}, \times\right)$, then the left adjoint $(-)^{\text {gpd }}:$ Cat $_{\infty} \rightarrow \mathcal{S}$ commutes with finite products, and hence a natural transformation $[1] \times \mathcal{C} \rightarrow \mathcal{D}$ gives rise to a map $([1] \times \mathcal{C})^{\mathrm{gpd}} \simeq[1]^{\text {gpd }} \times \mathcal{C}^{\mathrm{gpd}} \rightarrow \mathcal{D}^{\text {gpd }}$ which selects the desired equivalence in $\operatorname{hom}_{\mathcal{S}}\left(\operatorname{egpd}^{\mathrm{gpd}}, \mathcal{D}^{\mathrm{gpd}}\right)$.

In turn, Lemma 1.26 has the following useful further special case.

Corollary 1.28. An adjunction $F: \mathcal{C} \rightleftarrows \mathcal{D}: G$ induces inverse equivalences $F^{\mathrm{gpd}}:$ Cgpd $^{\sim} \mathcal{D}^{\text {gpd }}$ and $\mathcal{C}^{\text {gpd }} \stackrel{\sim}{\sim} \mathcal{D}^{\text {gpd }}: G^{\text {gpd }}$ in $\mathcal{S}$.

Proof. The adjunction $F \dashv G$ has unit and counit natural transformations ide $\rightarrow G \circ F$ and $F \circ G \rightarrow \operatorname{id}_{\mathcal{D}}$, and so the claim follows from Lemma 1.26.

We note the following interaction between taking localizations and taking homotopy categories.

Remark 1.29. Observe that the composite left adjoint

$$
\text { RelCat }_{\infty} \stackrel{(\text { ho }(-), \text { ho( }(-))}{\longrightarrow} \text { RelCat } \stackrel{(-)\left[(-)^{-1}\right]}{\longrightarrow} \text { Cat }
$$

coincides with the composite left adjoint

$$
\operatorname{RelCat}_{\infty} \stackrel{(-) \llbracket(-)^{-1} \rrbracket}{\longrightarrow} \text { Cat }_{\infty} \stackrel{\text { ho }}{\longrightarrow} \text { Cat }
$$

since they share a right adjoint

$$
\text { RelCat }_{\infty} \hookleftarrow \text { RelCat } \stackrel{\min }{\longleftarrow} \text { Cat. }
$$

Hence, for any $(\mathcal{R}, \mathbf{W}) \in \mathcal{R e l C a t}_{\infty}$ we have a natural equivalence

$$
\mathrm{ho}\left(\mathcal{R} \llbracket \mathbf{W}^{-1} \rrbracket\right) \stackrel{\sim}{\rightarrow} \operatorname{ho}(\mathcal{R})\left[\mathrm{ho}(\mathbf{W})^{-1}\right]
$$

in Cat $\subset$ Cat $_{\infty}$.

We end this section with the following observation (which partly echoes Example S.2.11). 
Remark 1.30. Suppose $(\mathcal{R}, \mathbf{W})$ is a relative $\infty$-category. Then $(\mathrm{ho}(\mathcal{R}), \mathrm{ho}(\mathbf{W}))$ is a relative category (so is in particular a relative $\infty$-category). However, its localization ho( $(\mathcal{R}) \llbracket$ ho $(\mathbf{W})^{-1} \rrbracket$ need not recover $\mathcal{R} \llbracket \mathbf{W}^{-1} \rrbracket$. This is for the same reason as such facts always are, namely that we lose coherence data when we pass from $\mathcal{R}$ to ho $(\mathcal{R})$. (Commutative diagrams in ho $(\mathcal{R})$ need not come from commutative diagrams in $\mathcal{R}$, and when they do they might do so in multiple, inequivalent ways.) An explicit counterexample is provided by the minimal relative $\infty$-category $(\mathcal{R}, \mathbf{W})=(\mathcal{R}, \mathcal{R} \simeq)$ : then

$$
\operatorname{ho}(\mathbf{W}) \simeq h_{0}\left(\mathcal{R}^{\simeq}\right) \simeq h_{0}(\mathcal{R}) \simeq \subset \operatorname{ho}(\mathcal{R})
$$

since the equivalences in $\mathcal{R}$ are created by $\mathcal{R} \rightarrow$ ho $(\mathcal{R})$, and hence ho $(\mathcal{R}) \llbracket$ ho $(\mathbf{W})^{-1} \rrbracket \simeq$ ho $(\mathcal{R})$ (while of course $\left.\mathcal{R} \llbracket \mathbf{W}^{-1} \rrbracket \simeq \mathcal{R}\right)$. One might therefore refer to the $\infty$-category ho $(\mathcal{R}) \llbracket$ ho $(\mathbf{W})^{-1} \rrbracket$ as an "exotic enrichment" of the homotopy category ho $\left(\mathcal{R} \llbracket \mathbf{W}^{-1} \rrbracket\right)$.

\section{Complete Segal SPaces}

We now give an extremely brief review of the theory of complete Segal spaces. This section exists moreor-less solely to fix notation; we refer the reader seeking a more thorough discussion either to the original paper [Rez01] (which uses model categories) or to [Lur09b, §1] (which uses $\infty$-categories).

Let us write $\boldsymbol{\Delta} \stackrel{[\bullet]}{\longrightarrow}$ Cat for the standard cosimplicial category. Then, recall that the nerve of a category $\mathcal{C}$ is the simplicial set $\mathrm{N}(\mathcal{C}) \bullet=\operatorname{hom}_{\mathcal{C a t}}^{\mathrm{lw}}([\bullet], \mathcal{C})$. This defines a fully faithful embedding $\mathrm{N}: \mathcal{C}$ at $\rightarrow s \mathcal{S e t}$, with image those simplicial sets which admit unique lifts for the inner horn inclusions $\left\{\Lambda_{i}^{n} \rightarrow \Delta^{n}\right\}_{0<i<n \geq 0}$. In fact, this functor is a right adjoint.

The situation with $\infty$-categories is completely analogous.

Definition 2.1. The ( $\infty$-categorical) nerve of an $\infty$-category $\mathcal{C}$ is the simplicial space

$$
\mathrm{N}_{\infty}(\mathcal{C}) \bullet=\operatorname{hom}_{\mathcal{C a t}_{\infty}}^{1 \mathrm{w}}([\bullet], \mathcal{C})
$$

i.e. the composite

$$
\boldsymbol{\Delta}^{o p} \stackrel{[\bullet]^{o p}}{\longrightarrow} \text { Cat }^{o p} \hookrightarrow\left(\text { Cat }_{\infty}\right)^{o p} \stackrel{\text { hom }_{\mathrm{Cat}_{\infty}}(-, \mathcal{C})}{\longrightarrow} \mathcal{S} .
$$

This defines a fully faithful embedding $\mathrm{N}_{\infty}: \operatorname{Cat}_{\infty} \hookrightarrow s \mathcal{S}$, with image the full subcategory $\mathcal{C S S} \subset s \mathcal{S}$ of complete Segal spaces, i.e. those simplicial spaces satisfying the Segal condition and the completeness condition. This inclusion fits into a left localization adjunction $\mathrm{L}_{\mathcal{C S S}}: s \mathcal{S} \rightleftarrows$ CSS $: \mathrm{U}_{\mathcal{E S S}}$. Hence, we obtain an equivalence

$$
\operatorname{Cat}_{\infty} \stackrel{\mathrm{N}_{\infty}}{\sim} \text { eSS }
$$

whose inverse

$$
\operatorname{eSS} \stackrel{\mathrm{N}_{\infty}^{-1}}{\sim} \operatorname{Cat}_{\infty}
$$

takes an object $Y_{\bullet} \in \mathcal{E S S}$ to the coend

$$
\int^{[n] \in \Delta} Y_{n} \times[n]
$$

in Cat $_{\infty}$. (These claims respectively follow from Proposition A.A.7.10], [JT07, Theorem 4.12], [Rez01, Theorem 7.2], and [JT07, Theorem 4.12] again.) This equivalence identifies subcategory $\mathcal{S} \subset$ Cat $_{\infty}$ with the subcategory of constant simplicial spaces (which are automatically complete Segal spaces).

Remark 2.2. Complete Segal spaces provide an extremely efficient way of computing the hom-spaces in an $\infty$-category: if $x, y \in \mathcal{C}$, then there is a natural equivalence

$$
\operatorname{hom}_{\mathcal{C}}(x, y) \simeq \lim \left(\begin{array}{c}
\mathrm{N}_{\infty}(\mathcal{C})_{1} \\
\underset{\downarrow}{\mid}(s, t) \\
\operatorname{pt}_{\mathcal{S}} \underset{(x, y)}{\longrightarrow} \mathrm{N}_{\infty}(\mathcal{C})_{0} \times \mathrm{N}_{\infty}(\mathcal{C})_{0}
\end{array}\right)
$$

in $\mathcal{S}$, where we use the notations $s=\delta_{1}$ and $t=\delta_{0}$ to emphasize the roles that these two face maps play in this theory. (Note that $\mathrm{N}_{\infty}(\mathcal{C})_{0}=$ hom $_{\mathcal{C}_{\infty}}([0], \mathcal{C}) \simeq \mathfrak{C} \simeq$ is simply the maximal subgroupoid of $\mathfrak{C}$, while $\mathrm{N}_{\infty}(\mathcal{C})_{1}=\operatorname{hom}_{\mathcal{C a t}_{\infty}}([1], \mathcal{C}) \simeq \operatorname{Fun}([1], \mathcal{C}) \simeq$ is the space morphisms in $\mathcal{C}$. $)$ 
Remark 2.3. There is a canonical involution $\Delta \stackrel{\sim}{\rightarrow} \boldsymbol{\Delta}$ in eat, which is the identity on objects but acts on morphisms by "reversing the coordinates": a map $[m] \stackrel{\varphi}{\rightarrow}[n]$ is taken to the map

$$
[m] \stackrel{i \mapsto(n-\varphi(m-i))}{\longrightarrow}[n] .
$$

Taking opposites, this induces an involution $\boldsymbol{\Delta}^{o p} \stackrel{\sim}{\rightarrow} \boldsymbol{\Delta}^{o p}$, which in turn induces an involution of $s \mathcal{S}=$ $\operatorname{Fun}\left(\boldsymbol{\Delta}^{o p}, \mathcal{S}\right)$ by precomposition. Unwinding the definitions, we see that this involution $s \mathcal{S} \stackrel{\sim}{\rightarrow} s \mathcal{S}$ restricts to an involution $\mathrm{CSS} \stackrel{\sim}{\rightarrow}$ CSS which corresponds to the involution $(-)^{o p}:$ Cat $_{\infty} \stackrel{\sim}{\rightarrow}$ Cat $_{\infty}$.

For future use, we record the following observation.

Proposition 2.4. The diagram

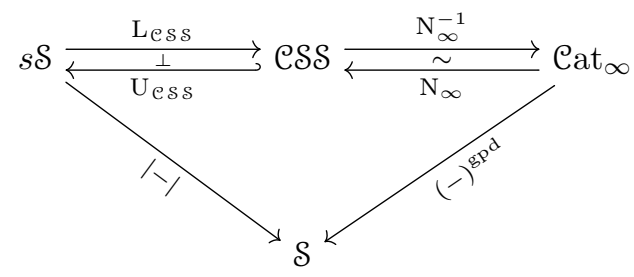

commutes: that is,

- geometric realization of complete Segal spaces models groupoid completion of $\infty$-categories, and

- for any $Y \in s \mathcal{S}$, the localization map $Y \rightarrow \mathrm{L}_{\mathcal{C s s}}(Y)$ becomes an equivalence upon geometric realization.

Proof. For the first claim, note that the functor $(-)^{\text {gpd }}:$ Cat $_{\infty} \rightarrow \mathcal{S}$ is a left localization, and the composite

$$
\mathcal{S} \stackrel{\mathrm{U}_{\mathrm{s}}}{\longrightarrow} \mathrm{Cat}_{\infty} \stackrel{\mathrm{N}_{\infty}}{\sim} \text { eSS } \stackrel{\mathrm{U}_{\text {ess }}}{\longrightarrow} s \mathcal{S}
$$

agrees with the functor const $: \mathcal{S} \rightarrow s \mathcal{S}$. Hence, the equivalence

$$
|-| \circ \mathrm{U}_{\mathcal{e s S}} \circ \mathrm{N}_{\infty} \simeq(-)^{\mathrm{gpd}}
$$

in $\operatorname{Fun}\left(\operatorname{Cat}_{\infty}, \mathcal{S}\right)$ follows from the uniqueness of left adjoints.

For the second claim, note that the reflective inclusion const $: \mathcal{S} \hookrightarrow s \mathcal{S}$ factors through the reflective inclusion UeSS $: \mathcal{C S S} \hookrightarrow s \mathcal{S}$. Hence, the factorization $\mathcal{S} \hookrightarrow \mathcal{C S S}$ is also a reflective inclusion. The equivalence

$$
|-| \simeq|-| \circ \mathrm{U}_{\text {ess }} \circ \mathrm{L}_{\mathrm{ess}}
$$

in $\operatorname{Fun}(s \mathcal{S}, \mathcal{S})$ now also follows from the uniqueness of left adjoints.

Remark 2.5. We may interpret Proposition 2.4 as saying that, while a simplicial space $Y \in s \mathcal{S}$ can be thought of as generating an $\infty$-category (namely the one corresponding to $\operatorname{L}_{\mathcal{C S S}}(Y) \in \mathcal{C S S}$ ), we can already directly extract its groupoid completion from $Y$ itself. This is analogous to the fact that an arbitrary simplicial set can be thought of as generating a quasicategory via fibrant replacement in $s \mathcal{S e t}_{\text {Joyal }}$, and the replacement map lies in $\mathbf{W}_{\text {Joyal }} \subset \mathbf{W}_{\mathrm{KQ}}$ (i.e. it induces an equivalence on geometric realizations).

Remark 2.6. Given a strict category $\mathcal{C} \in$ eat, the maps $\operatorname{hom}_{\text {eat }}([n], \mathcal{C}) \rightarrow$ hom $_{\mathcal{C}^{2}}([n], \mathcal{C})$ from hom-sets to hom-spaces collect into a map

$$
\mathrm{N}(\mathfrak{C}) \rightarrow \mathrm{N}_{\infty}(\mathfrak{C})
$$

in $s \mathcal{S}$; in turn, these maps assemble into a natural transformation $\mathrm{N} \rightarrow \mathrm{N}_{\infty}$ in Fun(eat, $s \mathcal{S}$ ). This map will be an equivalence in $s \mathcal{S}$ if and only if $\mathcal{C}$ is gaunt: while the nerve $\mathrm{N}(\mathcal{C}) \in s \mathcal{S}$ et $\subset s \mathcal{S}$ is always a Segal space, it only satisfies the completeness condition when every isomorphism in $\mathcal{C}$ is actually an identity map. ${ }^{7}$ However, by [Rez01, Remark 7.8], the above map induces an equivalence

$$
\mathrm{Less}_{\operatorname{es}}(\mathrm{N}(\mathcal{C})) \stackrel{\sim}{\rightarrow} \mathrm{Less}_{\operatorname{Cos}}\left(\mathrm{N}_{\infty}(\mathcal{C})\right) \simeq \mathrm{N}_{\infty}(\mathcal{C})
$$

in $\mathcal{S S S} \subset s \mathcal{S}$. In particular, it therefore follows from Proposition 2.4 that it also induces an equivalence

$$
|\mathrm{N}(\mathrm{C})| \stackrel{\sim}{\rightarrow}\left|\mathrm{N}_{\infty}(\mathcal{C})\right|
$$

in $\mathcal{S}$.

\footnotetext{
${ }^{7}$ Note that the Segal condition in $s \mathcal{S}$ et can be equivalently checked in $s \mathcal{S}$ since the inclusion $s \mathcal{S}$ et $\subset s \mathcal{S}$ is a right adjoint.
} 


\section{THE REZK NERVE}

Recall that the localization of a relative $\infty$-category $(\mathcal{R}, \mathbf{W})$ is the initial $\infty$-category $\mathcal{R} \llbracket \mathbf{W}^{-1} \rrbracket$ equipped with a functor from $\mathcal{R}$ which sends the subcategory $\mathbf{W} \subset \mathcal{R}$ of weak equivalences to equivalences. Meanwhile, given an arbitrary $\infty$-category $\mathcal{C}$, observe that the $n^{\text {th }}$ space of its nerve can be considered as

$$
\mathrm{N}_{\infty}(\mathcal{C})_{n}=\operatorname{hom}_{\mathcal{C a t}_{\infty}}([n], \mathcal{C}) \simeq \operatorname{Fun}([n], \mathcal{C}) \simeq \subset \operatorname{Fun}([n], \mathcal{C}),
$$

the subcategory of $\operatorname{Fun}([n], \mathcal{C})$ whose morphisms are the natural equivalences. Putting these two facts together, one is led to suspect that the $n^{\text {th }}$ space of the nerve $\mathrm{N}_{\infty}\left(\mathcal{R} \llbracket \mathbf{W}^{-1} \rrbracket\right)$ • should somehow contain the subcategory

$$
\operatorname{Fun}([n], \mathcal{R})^{\mathbf{W}} \subset \operatorname{Fun}([n], \mathcal{R})
$$

of $\operatorname{Fun}([n], \mathcal{R})$ whose morphisms are the natural weak equivalences. Of course, this will not generally form a space, but will instead be an $\infty$-category. On the other hand, there is a universal choice for a space admitting a map from this $\infty$-category, namely its groupoid completion. We are thus naturally led to make the following construction, a direct generalization of the "classification diagram" construction for relative categories defined in [Rez01, 3.3].

Definition 3.1. Given a relative $\infty$-category $(\mathcal{R}, \mathbf{W})$, its ( $\infty$-categorical) $\boldsymbol{R}$ ezk pre-nerve is the simplicial $\infty$-category

i.e. the composite

$$
\operatorname{preN}_{\infty}^{\mathrm{R}}(\mathcal{R}, \mathbf{W}) \bullet=\operatorname{Fun}^{1 \mathrm{w}}([\bullet], \mathcal{R})^{\mathbf{W}},
$$

$$
\boldsymbol{\Delta}^{o p} \stackrel{[\bullet]^{o p}}{\longrightarrow} \operatorname{Cat}^{o p} \hookrightarrow\left(\mathrm{Cat}_{\infty}\right)^{o p} \stackrel{\min ^{o p}}{\longrightarrow}\left(\mathcal{R e l e a t}_{\infty}\right)^{o p} \stackrel{\text { Fun }(-, \mathcal{R})^{\mathrm{W}}}{\longrightarrow} \operatorname{Cat}_{\infty}
$$

This defines a functor

$$
\operatorname{Releat}_{\infty} \stackrel{\operatorname{preN}_{\infty}^{\mathrm{R}}}{\longrightarrow} s \mathrm{Cat}_{\infty} .
$$

Then, the ( $\infty$-categorical) Rezk nerve functor

$$
\operatorname{RelCat}_{\infty} \stackrel{\mathrm{N}_{\infty}^{\mathrm{R}}}{\longrightarrow} s \mathcal{S}
$$

is given by the composite

$$
\operatorname{RelCat}_{\infty} \stackrel{\operatorname{preN}_{\infty}^{\mathrm{R}}}{\longrightarrow} s \mathrm{Cat}_{\infty} \stackrel{s(-)^{\mathrm{gpd}}}{\longrightarrow} s \mathcal{S} .
$$

Remark 3.2. Recall that Rezk's "classification diagram" construction of [Rez01, 3.3], which we will denote by

$$
\text { Releat } \stackrel{\mathrm{N}^{\mathrm{R}}}{\longrightarrow} s(s \mathcal{S e t})
$$

and refer to as the 1-categorical Rezk nerve functor, is given by the formula

$$
\mathrm{N}^{\mathrm{R}}(\mathcal{R}, \mathbf{W}) \bullet=\mathrm{N}\left(\operatorname{Fun}^{\mathrm{lw}}([\bullet], \mathcal{R})^{\mathbf{W}}\right) .
$$

Of course, we would like to think of this as a simplicial space using the model category $s\left(s \mathcal{S e t}_{\mathrm{KQ}}\right)_{\text {Reedy }}$. Indeed, combining Proposition 2.4 and Remark 2.6, we obtain a canonical commutative diagram

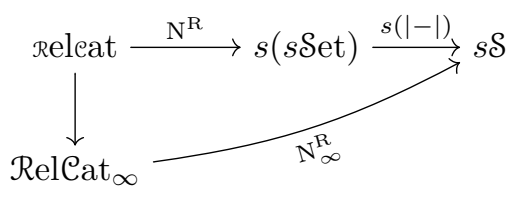

in $\mathrm{Cat}_{\infty}$; in fact, this even refines to a canonical commutative diagram

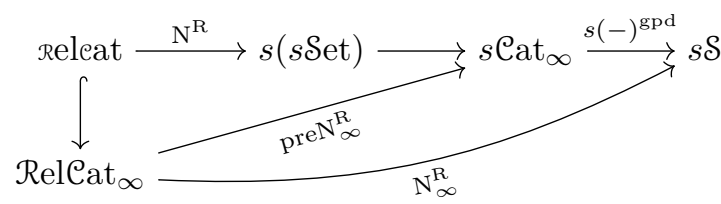

in $\operatorname{Cat}_{\infty}$ (in which the functor $s(s \mathcal{S e t}) \rightarrow s \mathrm{Cat}_{\infty}$ is obtained by applying $s(-)=\operatorname{Fun}\left(\boldsymbol{\Delta}^{o p},-\right.$ ) to the localization $\left.s \mathcal{S e t} \rightarrow s \operatorname{Set} \llbracket \mathbf{W}_{\text {Joyal }}^{-1} \rrbracket \simeq \mathcal{C a t}_{\infty}\right)$. Thus, at least as far as homotopical content is concerned, the $\infty$-categorical Rezk nerve functor strictly generalizes its 1-categorical counterpart. 
Remark 3.3. In turn, the 1-categorical Rezk nerve functor of Remark 3.2 suggests a similar model-dependent definition of a Rezk nerve functor for "marked quasicategories" (once again landing in $s s \mathcal{S}$ et). In fact, as the first step in the proof of Lemma 4.3, we will show that this construction is a model-categorical presentation

- of the $\infty$-categorical Rezk nerve when considered in $s\left(s \mathcal{S e t}_{\mathrm{KQ}}\right)_{\text {Reedy }}$, and in fact

- of the $\infty$-categorical Rezk pre-nerve when considered in $s\left(s\right.$ Set Joyal $_{\text {Reedy }}$.

Remark 3.4. We have the following slight reformulation of Definition 3.1: in view of Proposition 2.4, the Rezk nerve functor can also be described as a composite

$$
\text { RelCat }_{\infty} \stackrel{\text { preN }_{\infty}^{\mathrm{R}}}{\longrightarrow} s \mathrm{Cat}_{\infty} \simeq s \mathrm{CSS} \stackrel{s\left(\mathrm{U}_{\text {ess }}\right)}{\longrightarrow} s(s \mathcal{S}) \stackrel{s(|-|)}{\longrightarrow} s \mathcal{S} .
$$

Note that the composite functor $\mathcal{R e l C a t}_{\infty} \rightarrow s(s \mathcal{S})$ is a right adjoint, whose left adjoint is the left Kan extension

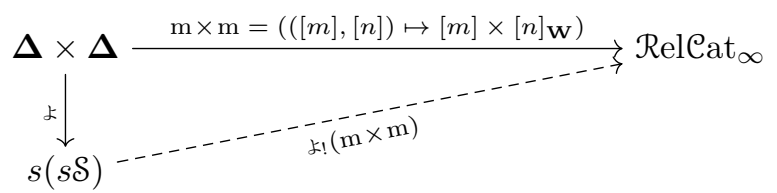

along the Yoneda embedding, where we write $m \times m$ for the upper "min $\times$ max" functor for brevity. On the other hand, the functor $s(|-|): s(s \mathcal{S}) \rightarrow s \mathcal{S}$ is a left adjoint. Hence, as the Rezk nerve functor is the composite of a right adjoint followed by a left adjoint, understanding its behavior in general is a rather difficult task. (In fact, it follows that $\operatorname{preN}_{\infty}^{\mathrm{R}}: \mathcal{R e l C a t}_{\infty} \rightarrow s \mathcal{C a t}_{\infty}$ is also a right adjoint, while $s(-)^{\text {gpd }}: s \mathcal{C a t}_{\infty} \rightarrow s \mathcal{S}$ is of course also a left adjoint.)

We have the following identifications of the Rezk nerves of minimal and maximal relative $\infty$-categories: in both of these extremal cases, the Rezk nerve does indeed compute the localization.

Proposition 3.5. The Rezk nerve functor acts on the full subcategories of RelCat ${ }_{\infty}$ spanned by the minimal and maximal relative $\infty$-categories (both of which can be indentified with Cat $_{\infty}$ ) according to the canonical commutative diagram

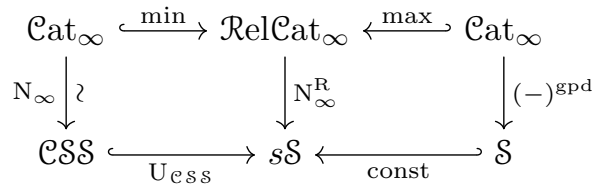

in $\mathrm{Cat}_{\infty}$.

Proof. To see that the left square commutes, given any $\mathcal{C} \in \mathrm{Cat}_{\infty}$ we compute that

$$
\operatorname{preN}_{\infty}^{\mathrm{R}}(\min (\mathcal{C}))_{n}=\operatorname{Fun}([n], \min (\mathcal{C}))^{\mathbf{W}} \simeq \operatorname{Fun}([n], \mathcal{C})^{\simeq} \simeq \operatorname{hom}_{\mathcal{C a t}_{\infty}}([n], \mathcal{C})=\mathrm{N}_{\infty}(\mathcal{C})_{n}
$$

(in a way compatible with the evident simplicial structure maps on both sides), i.e. we even have a canonical equivalence

$$
\operatorname{preN}_{\infty}^{\mathrm{R}}(\min (\mathcal{C})) \bullet \simeq \mathrm{N}_{\infty}(\mathcal{C}) \bullet
$$

in $s \mathrm{Cat}_{\infty}$. As $s(-)^{\text {gpd }}: s \mathcal{C a t}_{\infty} \rightleftarrows s \mathcal{S}: s\left(\mathrm{U}_{\mathcal{S}}\right)$ is a left localization adjunction, it follows that we also have a canonical equivalence

in $s \mathcal{S}$.

$$
\mathrm{N}_{\infty}^{\mathrm{R}}(\min (\mathcal{C})) \bullet \simeq \mathrm{N}_{\infty}(\mathcal{C})
$$

To see that the right square commutes, given any $\mathcal{C} \in \mathcal{C a t}_{\infty}$ we first compute that

$$
\operatorname{preN}_{\infty}^{\mathrm{R}}(\max (\mathcal{C}))_{n}=\operatorname{Fun}([n], \max (\mathcal{C}))^{\mathbf{W}} \simeq \operatorname{Fun}([n], \mathcal{C}) .
$$

Moreover, note that every face-then-degeneracy composite

$$
\operatorname{Fun}([n], \mathcal{C}) \stackrel{\delta_{i}}{\rightarrow} \operatorname{Fun}([n-1], \mathcal{C}) \stackrel{\sigma_{j}}{\longrightarrow} \operatorname{Fun}([n], \mathcal{C})
$$

admits a natural transformation either to or from $\operatorname{id}_{\mathrm{Fun}([n], \mathcal{e})}$ (depending on $i$ and $j$ ). ${ }^{8}$ By Lemma 1.26, it follows that all the structure maps of $\mathrm{N}_{\infty}^{\mathrm{R}}(\max (\mathcal{C})) \in s \mathcal{S}$ are equivalences, and hence (since $\boldsymbol{\Delta}^{o p}$ is sifted so

\footnotetext{
${ }^{8}$ We refer the reader to Lemma H.3.5 for a more general statement (whose proof of course does not rely on the present discussion in any way).
} 
in particular $\left.\left(\boldsymbol{\Delta}^{o p}\right)^{\mathrm{gpd}} \simeq \mathrm{pt}_{\mathcal{S}}\right)$ it follows that this simplicial space is constant. The commutativity of the right square now follows from the computation

$$
\mathrm{N}_{\infty}^{\mathrm{R}}(\max (\mathcal{C}))_{0}=\left(\operatorname{Fun}([0], \max (\mathcal{C}))^{\mathbf{W}}\right)^{\mathrm{gpd}} \simeq \mathfrak{C}^{\mathrm{gpd}},
$$

which gives rise to a canonical equivalence $\mathrm{N}_{\infty}^{\mathrm{R}}(\max (\mathcal{C})) \bullet \simeq \operatorname{const}\left(\mathcal{C}^{\text {gpd }}\right) \simeq \mathrm{N}_{\infty}\left(\mathcal{C}^{\text {gpd }}\right) \bullet$ in $s \mathcal{S}$.

Now, recall that any relative $\infty$-category $(\mathcal{R}, \mathbf{W})$ admits a natural map $\min (\mathcal{R})=(\mathcal{R}, \mathcal{R} \simeq) \rightarrow(\mathcal{R}, \mathbf{W})$ (namely the unit of the adjunction min $\dashv \mathrm{U}_{\mathcal{R e l}}$ ). Hence, by Proposition 3.5 we obtain a natural map

$$
\mathrm{N}_{\infty}(\mathcal{R}) \rightarrow \mathrm{N}_{\infty}^{\mathrm{R}}(\mathcal{R}, \mathbf{W})
$$

in $s \mathcal{S} .^{9}$ This immediately suggests the following two questions.

Question 3.6. When does this map in $s \mathcal{S}$ (or equivalently, its target) actually lie in the full subcategory $\mathcal{C S S} \subset s \mathcal{S}$ ?

Question 3.7. In light of the composite adjunction awhat is the $\infty$-categorical significance of this map?

We give a partial answer to Question 3.6 in [MGd] (see the calculus theorem (H.5.1)). Meanwhile, the essence of the present paper consists in the following complete answer to Question 3.7, the local universal property of the Rezk nerve.

Theorem 3.8. For any $(\mathcal{R}, \mathbf{W}) \in \mathcal{R e l C a t}_{\infty}$ and any $\mathcal{C} \in \mathcal{C a t}_{\infty}$, we have a commutative square

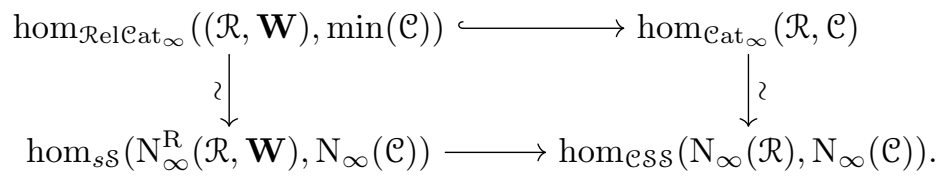

In other words, the natural map

$$
\mathrm{N}_{\infty}(\mathcal{R}) \simeq \mathrm{L}_{\mathcal{C S S}}\left(\mathrm{N}_{\infty}(\mathcal{R})\right) \rightarrow \mathrm{L}_{\mathcal{C S S}}\left(\mathrm{N}_{\infty}^{\mathrm{R}}(\mathcal{R}, \mathbf{W})\right)
$$

in SSS corresponds to the localization map $\mathcal{R} \rightarrow \mathcal{R} \llbracket \mathbf{W}^{-1} \rrbracket$ in Cat $_{\infty}$.

We will give a proof of Theorem 3.8 in $\S 4$.

Using Theorem 3.8 as input, we can now prove a statement which will easily imply the global universal property of the Rezk nerve (Corollary 3.12).

Proposition 3.9. The composite functor

$$
\operatorname{RelCat}_{\infty} \stackrel{\mathrm{N}_{\infty}^{\mathrm{R}}}{\longrightarrow} s \mathcal{S} \stackrel{\mathrm{L}_{\mathcal{C S s}}}{\longrightarrow} \mathrm{CSS} \simeq \mathrm{Cat}_{\infty}
$$

induces an equivalence

$$
\text { RelCat }_{\infty} \llbracket \mathbf{W}_{\mathrm{BK}}^{-1} \rrbracket \stackrel{\sim}{\rightarrow} \operatorname{Cat}_{\infty} .
$$

In the proof of Proposition 3.9, it will be convenient to have the following terminology.

Definition 3.10. We define the subcategory $\mathbf{W}_{\text {Rezk }} \subset s s \mathcal{S}$ of Rezk weak equivalences to be created by the composite

$$
s(s \mathcal{S}) \stackrel{s(|-|)}{\longrightarrow} s \mathcal{S} \stackrel{\mathrm{L}_{e s s}}{\longrightarrow} \mathcal{C S S} \simeq \mathrm{Cat}_{\infty} .
$$

(This name is meant to be suggestive of Rezk's "complete Segal space" model structure on the category ssSet of bisimplicial sets.) We denote the resulting relative $\infty$-category by $s s \mathcal{S}_{\text {Rezk }}=\left(s s \mathcal{S}, \mathbf{W}_{\text {Rezk }}\right) \in \mathcal{R e l C a t}_{\infty}$. Since left localizations are in particular free localizations (recall Example 1.13), this composite left adjoint induces an equivalence

$$
s s \mathcal{S} \llbracket \mathbf{W}_{\text {Rezk }}^{-1} \rrbracket \stackrel{\sim}{\longrightarrow} \operatorname{Cat}_{\infty}
$$

in $\mathrm{Cat}_{\infty}$.

\footnotetext{
${ }^{9}$ This can also be obtained from the levelwise inclusion $\operatorname{hom}_{\mathcal{C}^{\mathrm{w}}{ }_{\infty}}^{\mathrm{w}}([\bullet], \mathcal{R}) \simeq\left(\operatorname{Fun}^{\mathrm{lw}}([\bullet], \mathcal{R})^{\mathbf{W}}\right)^{\simeq} \hookrightarrow \operatorname{Fun}^{\mathrm{lw}}([\bullet], \mathcal{R})^{\mathbf{W}}$ of maximal subgroupoids.
} 
Proof of Proposition 3.9. Recalling Remark 3.4, we have a composite adjunction

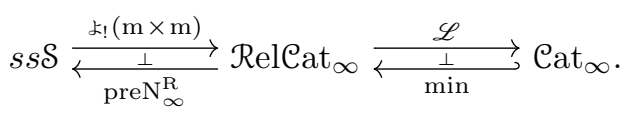

Moreover, it follows from Proposition 3.5 that the right adjoint of this composite adjunction is precisely that of the composite adjunction

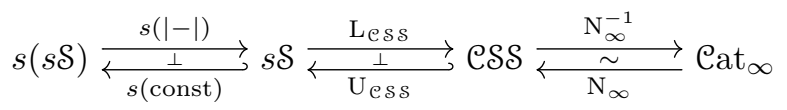

whose left adjoint defines $\mathbf{W}_{\text {Rezk }} \subset s s \mathcal{S}$, and hence in particular it follows that the right adjoint of our original composite adjunction defines a weak equivalence

$$
s s \mathcal{S}_{\text {Rezk }} \stackrel{\operatorname{minopreN} \mathrm{N}_{\infty}^{\mathrm{R}}}{\approx} \min \left(\text { Cat }_{\infty}\right)
$$

in $\left(\text { RelCat }_{\infty}\right)_{\mathrm{BK}}$.

Next, we claim that the right adjoint $\mathcal{R e l C a t}_{\infty} \stackrel{\operatorname{preN}_{\infty}^{\mathrm{R}}}{\longrightarrow} s s \mathcal{S}$ is a relative functor. To see this, first note that given any $(\mathcal{R}, \mathbf{W}) \in \mathcal{R e l C a t}_{\infty}$, we obtain a counit map

$$
(\mathcal{R}, \mathbf{W}) \stackrel{\approx}{\rightarrow} \min \left(\mathcal{R} \llbracket \mathbf{W}^{-1} \rrbracket\right)
$$

in $\left(\mathcal{R e l C a t}_{\infty}\right)_{\mathrm{BK}}$ from the adjunction $\mathscr{L} \dashv$ min. Theorem 3.8 and Proposition 3.5 then together imply that applying the functor $\mathcal{R e l C a t}_{\infty} \stackrel{\text { preN }_{\infty}^{\mathrm{R}}}{\longrightarrow} s s \mathcal{S}$ to this map yields a weak equivalence

$$
\operatorname{preN}_{\infty}^{\mathrm{R}}(\mathcal{R}, \mathbf{W}) \stackrel{\widetilde{\rightarrow}}{\operatorname{preN}} \operatorname{pr}_{\infty}^{\mathrm{R}}\left(\min \left(\mathcal{R} \llbracket \mathbf{W}^{-1} \rrbracket\right)\right) \simeq \operatorname{const}^{\mathrm{lw}}\left(\mathrm{N}_{\infty}\left(\mathcal{R} \llbracket \mathbf{W}^{-1} \rrbracket\right)\right)
$$

in $s s \mathcal{S}_{\text {Rezk }}$. Hence, any weak equivalence $\left(\mathcal{R}_{1}, \mathbf{W}_{1}\right) \stackrel{\widetilde{\rightarrow}}{\rightarrow}\left(\mathcal{R}_{2}, \mathbf{W}_{2}\right)$ in $\left(\text { RelCat }_{\infty}\right)_{\text {BK }}$ induces a commutative diagram

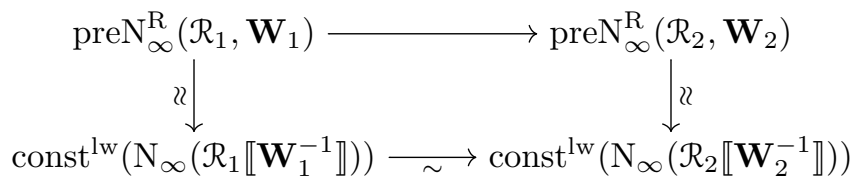

in $s s \mathcal{S}_{\text {Rezk }}$, and then the top arrow in this square is also in $\mathbf{W}_{\text {Rezk }} \subset s s \mathcal{S}$ since it has the two-out-of-three property. So this does indeed define a relative functor

$$
\left(\mathcal{R e l e a t}_{\infty}\right)_{\mathrm{BK}} \stackrel{\operatorname{preN}_{\infty}^{\mathrm{R}}}{\longrightarrow} s s \mathcal{S}_{\text {Rezk }} .
$$

From here, it follows that the right adjoints of our original composite adjunction form a commutative diagram

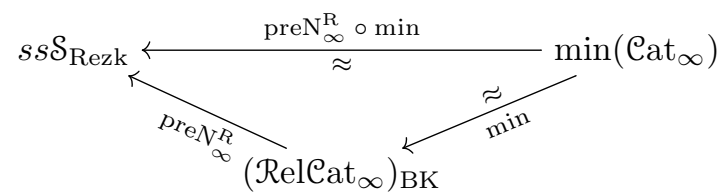

in $\left(\mathcal{R e l C a t}_{\infty}\right)_{\mathrm{BK}}$, and so the entire diagram lies in $\mathbf{W}_{\mathrm{BK}} \subset \mathcal{R}$ elCat ${ }_{\infty}$ since it has the two-out-of-three property. Hence, we obtain a commutative diagram

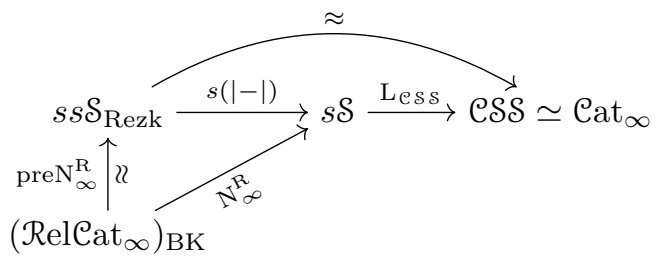

in $\left(\mathcal{R e l C a t}_{\infty}\right)_{\mathrm{BK}}$, which proves the claim. 
Remark 3.11. It does not appear possible to give a completely hands-off proof of Proposition 3.9, i.e. one not relying on Theorem 3.8 (or perhaps even one that would prove Theorem 3.8 as a formal consequence). More specifically, adjunctions of underlying $\infty$-categories do not necessarily play well with relative $\infty$-category structures, even if one of the adjoints is a relative functor: one must have some control over the behavior of both adjoints.

For instance, the geometric realization functor $s \mathcal{S} \stackrel{|-|}{\longrightarrow} \mathcal{S}$ and its restriction to the subcategory $s \mathcal{S}$ et $\subset$ $s \mathcal{S}$ create subcategories of weak equivalences which define the Kan-Quillen relative $\infty$-category structures $\left(s \mathcal{S}, \mathbf{W}_{\mathrm{KQ}}^{s \mathcal{S}}\right),\left(s \mathcal{S e t}, \mathbf{W}_{\mathrm{KQ}}^{s \mathcal{S e t}}\right) \in \mathcal{R}^{\text {RelCat }}{ }_{\infty}$ (which underlie their respective Kan-Quillen model structures (see $\S$ S.4)). Moreover, these relative $\infty$-categories give rise to a diagram

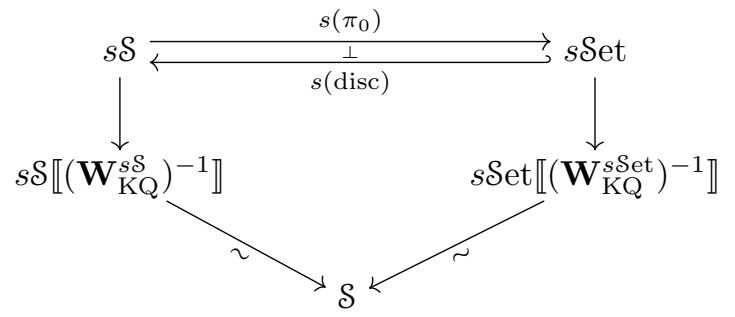

in which the right adjoint commutes with the respective localization functors: in other words, it induces a weak equivalence

$$
\left(s \mathcal{S}_{\mathrm{KQ}}, \mathbf{W}_{\mathrm{KQ}}^{s \mathcal{S}}\right) \approx\left(s \mathcal{S e t}_{\mathrm{KQ}}, \mathbf{W}_{\mathrm{KQ}}^{s \mathcal{S} \mathrm{t}}\right)
$$

in $\left(\mathcal{R e l C a t}_{\infty}\right)_{\mathrm{BK}}$. Nevertheless, the left adjoint is clearly very far from also defining a weak equivalence in $\left(\mathcal{R e l e a t}_{\infty}\right)_{\mathrm{BK}}$.

We can now prove the global universal property of the Rezk nerve.

Corollary 3.12. The composite functor

$$
\text { RelCat }_{\infty} \stackrel{\mathrm{N}_{\infty}^{\mathrm{R}}}{\longrightarrow} s \mathcal{S} \stackrel{\mathrm{L}_{\mathcal{C S S}}}{\longrightarrow} \text { CSS } \stackrel{\mathrm{N}_{\infty}^{-1}}{\sim} \text { Cat }_{\infty}
$$

is canonically equivalent in $\mathrm{Fun}\left(\mathcal{R e l e a t}_{\infty}, \mathrm{Cat}_{\infty}\right)$ to the localization functor

$$
\text { RelCat }_{\infty} \stackrel{\mathscr{L}}{\longrightarrow} \operatorname{Cat}_{\infty} .
$$

Proof. Since these functors both take the subcategory $\mathbf{W}_{\mathrm{BK}} \subset \mathcal{R e l C a t}_{\infty}$ into $\left(\right.$ Cat $\left._{\infty}\right) \simeq \subset$ Cat $_{\infty}$, they factor uniquely through the localization

$$
\text { RelCat }_{\infty} \rightarrow \text { RelCat }_{\infty} \llbracket \mathbf{W}_{\text {BK }}^{-1} \rrbracket .
$$

The resulting functors $\mathcal{R e l} \mathrm{Cat}_{\infty} \llbracket \mathbf{W}_{\mathrm{BK}}^{-1} \rrbracket \rightarrow$ Cat $_{\infty}$ are then both equivalences, the former by Proposition 3.9 and the latter by Proposition 1.17. The result now follows by inspection, using the fact that

$$
\operatorname{hom}_{\mathcal{C a t}_{\infty}}\left(\operatorname{Cat}_{\infty}, \operatorname{Cat}_{\infty}\right) \simeq \mathbb{Z} / 2
$$

(see [Toë05, Théorèm 6.3] or [Lur09b, Theorem 4.4.1]).

Remark 3.13. The global universal property of the Rezk nerve (Corollary 3.12) can be seen as a generalization of work of Barwick-Kan. To see this, consider the composite pair of Quillen adjunctions

$$
s\left(s \text { Set }_{\mathrm{KQ}}\right)_{\text {Reedy }} \rightleftarrows s s \mathcal{S e t} \text { Rezk } \rightleftarrows \text { Releat }_{\mathrm{BK}},
$$

where

- the first is the left Bousfield localization which defines the Rezk model structure (see [Rez01, Theorem 7.2]) and presents the adjunction $\mathrm{L}_{\mathcal{C S S}}: s \mathcal{S} \rightleftarrows \mathcal{C S S}: \mathrm{U}_{\mathcal{E S S}}$, and

- the second is the Quillen equivalence which defines the Barwick-Kan model structure (see [BK12, Theorem 6.1]).

As the latter is constructed using the lifting theorem for cofibrantly generated model categories, its right adjoint preserves all weak equivalences by definition. Moreover, Barwick-Kan provide a natural weak equivalence in $s\left(s \mathcal{S e t}_{\mathrm{KQ}}\right)_{\text {Reedy }}$ (and hence also in $s s \mathcal{S e t}_{\text {Rezk }}$ ) from the Rezk nerve functor to the right adjoint of their Quillen equivalence (see [BK12, Lemma 5.4]). 
Now, consider the commutative triangle

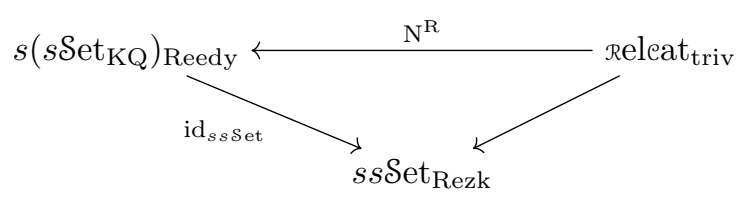

in RelCat (in which we take Releat with the trivial model structure since we are interested in relative categories themselves here). Applying the localization functor

$$
\text { RelCat } \hookrightarrow \text { RelCat }_{\infty} \stackrel{\mathscr{L}}{\longrightarrow} \text { Cat }_{\infty},
$$

this yields a commutative triangle

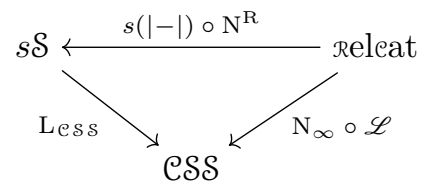

in $\mathrm{Cat}_{\infty}$, in which

- the upper map coincides with the composite

$$
\text { Releat } \rightarrow \text { Releat } \hookrightarrow \text { RelCat }_{\infty} \stackrel{\mathrm{N}_{\infty}^{\mathrm{R}}}{\longrightarrow} s \mathcal{S}
$$

by Remark 3.2, and

- the map releat $\rightarrow$ CSS can be identified as indicated since by what we have just seen it is equivalent to the projection

$$
\text { Releat } \rightarrow \text { Releat } \llbracket \mathbf{W}_{\mathrm{BK}}^{-1} \rrbracket \simeq \text { eat }_{\infty}
$$

to the underlying $\infty$-category (which is indeed given by localization).

It follows that we obtain a commutative diagram

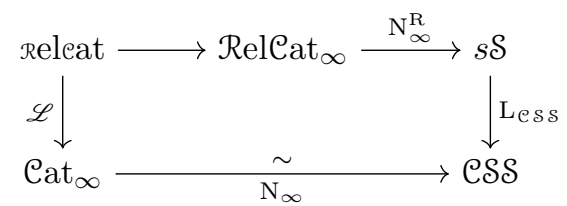

in Cat $_{\infty}$, which is precisely the restriction of the assertion of the global universal property of the Rezk nerve (Corollary 3.12) to the category releat, as claimed.

Remark 3.14. Taken together, Proposition 3.9 and Corollary 3.12 imply that in fact the adjunction

$$
s s \mathcal{S} \underset{\operatorname{preN}_{\infty}^{\mathrm{R}}}{\stackrel{\perp}{\stackrel{\perp}{L}}} \mathcal{R e l C a t}_{\infty}
$$

has

- that both adjoints are relative functors (with respect to their respective Rezk and Barwick-Kan relative structures), and

- that the unit and counit are both natural weak equivalences.

This can be seen as follows.

First of all, recall that in the proof of Proposition 3.9, we already saw that the right adjoint is a relative functor. On the other hand, the left adjoint is a relative functor because the composite left adjoint

$$
s s \mathcal{S} \stackrel{L_{!}(\mathrm{m} \times \mathrm{m})}{\longrightarrow} \operatorname{RelCat}_{\infty} \stackrel{\mathscr{L}}{\longrightarrow} \text { Cat }_{\infty}
$$

agrees with the left adjoint

$$
s s \mathcal{S} \stackrel{s(|-|)}{\longrightarrow} s \mathcal{S} \stackrel{\mathrm{L}_{\mathcal{C S S}}}{\longrightarrow} \operatorname{CSS} \stackrel{\mathrm{N}_{\infty}^{-1}}{\sim} \text { Cat }_{\infty}
$$

(since we have seen in the proof of Proposition 3.9 that they share a right adjoint), and so in fact the subcategory $\mathbf{W}_{\text {Rezk }} \subset s s \mathcal{S}$ is created by pulling back the subcategory $\mathbf{W}_{\mathrm{BK}} \subset$ Releat $_{\infty}$. 
Next, we can see that the counit map

$$
\downarrow_{!}(\mathrm{m} \times \mathrm{m})\left(\operatorname{preN}_{\infty}^{\mathrm{R}}(\mathcal{R}, \mathbf{W})\right) \rightarrow(\mathcal{R}, \mathbf{W})
$$

is a weak equivalence in $\left(\mathcal{R e l} \mathcal{C a t}_{\infty}\right)_{\mathrm{BK}}$ as follows. Applying the functor $\mathcal{R e l C a t}_{\infty} \stackrel{\mathscr{L}}{\longrightarrow} \operatorname{Cat}_{\infty}$, we obtain a map

$$
\mathscr{L}\left(\varsigma_{!}(\mathrm{m} \times \mathrm{m})\left(\operatorname{preN}_{\infty}^{\mathrm{R}}(\mathcal{R}, \mathbf{W})\right)\right) \rightarrow \mathcal{R} \llbracket \mathbf{W}^{-1} \rrbracket
$$

in $\mathcal{C a t}_{\infty}$. Then, again appealing to the fact that these composite left adjoints $s s \mathcal{S} \rightarrow$ Cat $_{\infty}$ agree, we can reidentify the source as

$$
\mathscr{L}\left(\Sigma_{!}(\mathrm{m} \times \mathrm{m})\left(\operatorname{preN}_{\infty}^{\mathrm{R}}(\mathcal{R}, \mathbf{W})\right) \simeq \mathrm{N}_{\infty}^{-1}\left(\operatorname{Less}_{\operatorname{Cs}}\left(s(|-|)\left(\operatorname{preN}_{\infty}^{\mathrm{R}}(\mathcal{R}, \mathbf{W})\right)\right)\right) \simeq \mathrm{N}_{\infty}^{-1}\left(\mathrm{~L}_{\mathcal{C} s s}\left(\mathrm{~N}_{\infty}^{\mathrm{R}}(\mathcal{R}, \mathbf{W})\right)\right) .\right.
$$

So, we can reidentify this map as

$$
\mathrm{N}_{\infty}^{-1}\left(\mathrm{~L}_{\operatorname{ess}}\left(\mathrm{N}_{\infty}^{\mathrm{R}}(\mathcal{R}, \mathbf{W})\right)\right) \rightarrow \mathcal{R} \llbracket \mathbf{W}^{-1} \rrbracket
$$

which is an equivalence by Theorem 3.8. So the counit map is indeed a weak equivalence in $\left(\mathcal{R e l} \mathrm{Cat}_{\infty}\right)_{\mathrm{BK}}$, i.e. the counit is a natural weak equivalence.

Finally, we can see that the unit map

$$
\operatorname{preN}_{\infty}^{\mathrm{R}}\left(\downarrow_{!}(\mathrm{m} \times \mathrm{m})(Y)\right) \rightarrow Y
$$

is a weak equivalence in $s s \mathcal{S}_{\text {Rezk }}$ as follows. Applying the composite left adjoint

$$
s s \mathcal{\mathrm { N } _ { \infty } ^ { - 1 } \circ \mathrm { L } _ { \mathcal { C } s s } \circ \mathrm { s } ( | - | )} \longrightarrow \mathrm{Cat}_{\infty}
$$

and appealing to Corollary 3.12 , we obtain a map

$$
\mathscr{L}(\text { よ! }(\mathrm{m} \times \mathrm{m})(Y)) \rightarrow \mathrm{N}_{\infty}^{-1}\left(\mathrm{~L}_{\operatorname{ess}}(s(|-|)(Y))\right)
$$

in $\mathrm{Cat}_{\infty}$, and the same equivalence of composite left adjoints $s s \mathcal{S} \rightarrow$ Cat $_{\infty}$ implies that this is an equivalence. So the unit map is indeed a weak equivalence in $s s \mathcal{S}_{\text {Rezk }}$, i.e. the unit is a natural weak equivalence.

\section{The PRoof of Theorem 3.8}

Let $(\mathcal{R}, \mathbf{W})$ be an arbitrary relative $\infty$-category. In this section, we show that as a simplicial space, its Rezk nerve $\mathrm{N}_{\infty}^{\mathrm{R}}(\mathcal{R}, \mathbf{W})$ enjoys the desired universal property for mapping into complete Segal spaces: for any $\mathcal{C} \in \mathcal{C a t}_{\infty}$, we have a commutative diagram

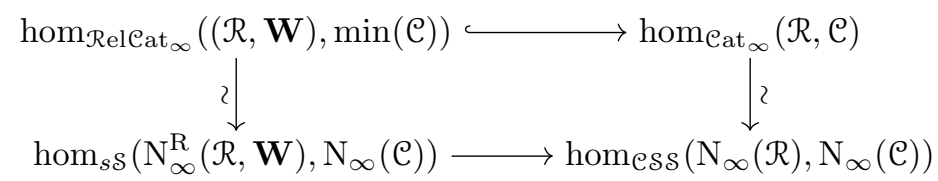

in $\mathcal{S}$, as asserted in Theorem 3.8 .

Most of the proof is reasonably straightforward, and we can give it immediately. But there will be one technical result (Lemma 4.3) that is necessary for the proof which will occupy us for the remainder of the section.

Proof of Theorem 3.8. By definition, the localization $\mathcal{R} \llbracket \mathbf{W}^{-1} \rrbracket \in$ Cat $_{\infty}$ is given as the pushout

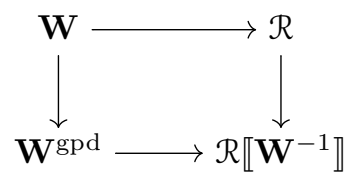

in Cat $_{\infty}$; under the equivalence $\mathrm{N}_{\infty}$ : Cat $_{\infty} \stackrel{\sim}{\rightarrow}$ ESS, this corresponds to a pushout diagram

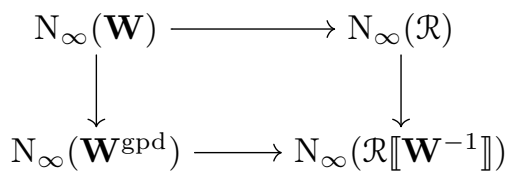


in $\mathcal{E S S} \subset s \mathcal{S}$. On the other hand, there is an evident commutative diagram

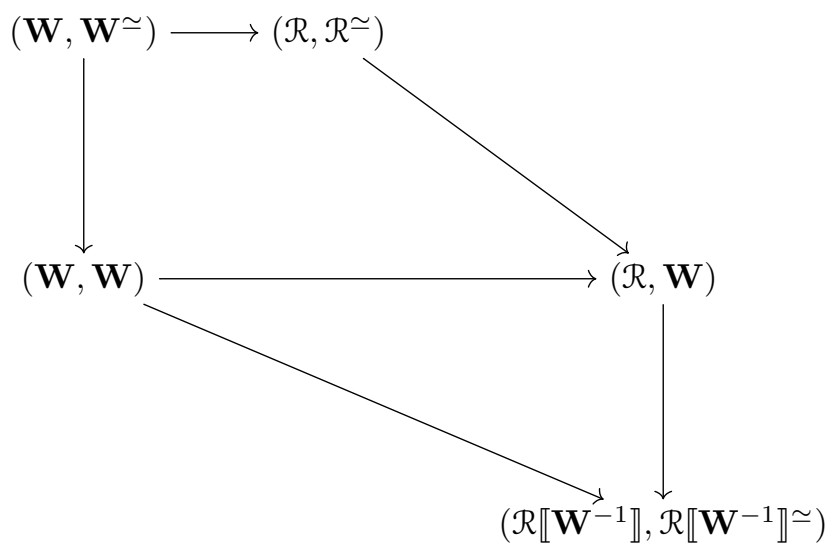

in RelCat $_{\infty}$. Applying the functor $\mathrm{N}_{\infty}^{\mathrm{R}}: \mathcal{R e l C a t}_{\infty} \rightarrow s \mathcal{S}$ and taking the pushout of the upper left span, in light of Proposition 3.5 we obtain a commutative diagram

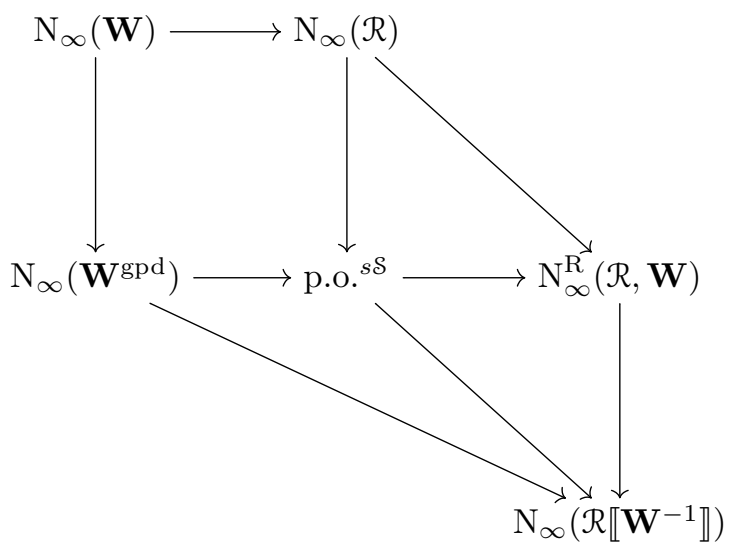

in $s \mathcal{S}$,

- where p.o. ${ }^{s \mathcal{S}}$ denotes the pushout in $s \mathcal{S}$ of the upper left span, and

- which contains as a subdiagram the above pushout square in $\mathcal{E S S} \subset s \mathcal{S}$.

Our goal is to prove that the induced map

$$
\mathrm{Less}_{\mathcal{C}}\left(\mathrm{N}_{\infty}^{\mathrm{R}}(\mathcal{R}, \mathbf{W})\right) \rightarrow \mathrm{L}_{\mathcal{e S S}}\left(\mathrm{N}_{\infty}\left(\mathcal{R} \llbracket \mathbf{W}^{-1} \rrbracket\right)\right) \simeq \mathrm{N}_{\infty}\left(\mathcal{R} \llbracket \mathbf{W}^{-1} \rrbracket\right)
$$

is an equivalence in $\mathcal{S S S} \subset s \mathcal{S}$.

For notational convenience, let us simply write

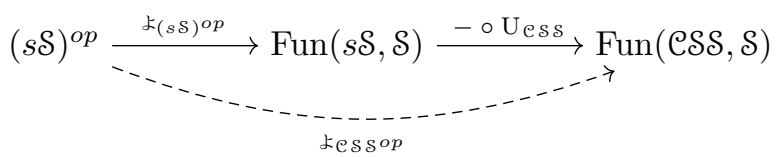

for the restricted contravariant Yoneda functor, so that for any $Y \in s \mathcal{S}$ we have

$$
\operatorname{Less}^{o p}(Y)=\operatorname{hom}_{s \mathcal{S}}\left(Y, \mathrm{U}_{\mathcal{C S S}}(-)\right) \simeq \operatorname{hom}_{\mathcal{C S S}}\left(\mathrm{L}_{\mathcal{C S S}}(Y),-\right)
$$

in Fun( $\mathcal{E S}, \mathcal{S})$. Then, by Yoneda's lemma, our aforestated goal is equivalent to proving that the map

$$
\mathrm{N}_{\infty}^{\mathrm{R}}(\mathcal{R}, \mathbf{W}) \rightarrow \mathrm{N}_{\infty}\left(\mathcal{R} \llbracket \mathbf{W}^{-1} \rrbracket\right)
$$

in $s \mathcal{S}$ induces an equivalence

$$
\operatorname{Lessop}^{o p}\left(\mathrm{~N}_{\infty}^{\mathrm{R}}(\mathcal{R}, \mathbf{W})\right) \leftarrow \operatorname{Lessop}\left(\mathrm{N}_{\infty}\left(\mathcal{R} \llbracket \mathbf{W}^{-1} \rrbracket\right)\right)
$$


in Fun(eSS, $\mathcal{S})$. Moreover, as the functor $s \mathcal{S} \stackrel{\mathrm{Less}}{\longrightarrow}$ CSS commutes with pushouts (being a left adjoint), it follows that the map

$$
\text { p.o. }^{s s} \rightarrow \mathrm{N}_{\infty}\left(\mathcal{R} \llbracket \mathbf{W}^{-1} \rrbracket\right)
$$

in $s \mathcal{S}$ induces an equivalence

$$
\mathrm{L}_{\text {ess }}\left(\text { p.o. }^{\text {ss }}\right) \stackrel{\sim}{\rightarrow} \mathrm{L}_{\mathcal{C s s}}\left(\mathrm{N}_{\infty}\left(\mathcal{R} \llbracket \mathbf{W}^{-1} \rrbracket\right)\right) \simeq \mathrm{N}_{\infty}\left(\mathcal{R} \llbracket \mathbf{W}^{-1} \rrbracket\right)
$$

in $\mathcal{C S S} \subset s \mathcal{S}$, and so the above diagram in $s \mathcal{S}$ gives rise to a retraction diagram

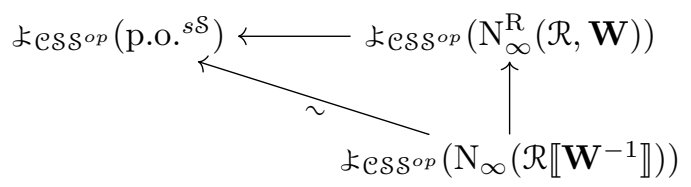

in Fun(eSS, $\mathcal{S})$ into which this map which we must show to be an equivalence fits, and which it therefore suffices to show is in fact a diagram of equivalences.

Now, observe that $\mathcal{S S S}$ is complete and hence in particular admits all cotensors, and observe moreover that the functor

$$
(s \mathcal{S})^{o p} \stackrel{\text { 上es } \mathcal{S}^{o p}}{\longrightarrow} \operatorname{Fun}(\mathrm{CSS}, \mathcal{S})
$$

factors through the contravariant Yoneda embedding and hence takes values in functors which commute with cotensors. So by Lemma 4.1, it suffices to show that after postcomposition with $\mathcal{S} \stackrel{\pi_{0}}{\longrightarrow} \mathcal{S}$ et, the above retraction diagram in Fun $(\mathcal{C S S}, \mathcal{S})$ becomes a diagram of natural isomorphisms in Fun(eSS, Set). Hence, it suffices to show that the induced map

$$
\left(\pi_{0} \circ \text { Lessop }\left(\mathrm{N}_{\infty}^{\mathrm{R}}(\mathcal{R}, \mathbf{W})\right)\right) \rightarrow\left(\pi_{0} \circ \text { Less } \mathcal{S}^{o p}\left(\text { p.o. }^{s \mathcal{S}}\right)\right)
$$

is a natural monomorphism in Fun(eSS, Set). This follows from the stronger statement that the composite

$$
\left(\pi_{0} \circ \text { Less'op }\left(\mathrm{N}_{\infty}^{\mathrm{R}}(\mathcal{R}, \mathbf{W})\right)\right) \rightarrow\left(\pi_{0} \circ \text { Less }{ }^{o p}\left(\text { p.o. }^{s \mathcal{S}}\right)\right) \rightarrow\left(\pi_{0} \circ \text { Lessop }\left(\mathrm{N}_{\infty}(\mathcal{R})\right)\right)
$$

is a natural monomorphism in Fun(eSS, Set), which in turn follows from Lemma 4.3.

We needed the following easy result in the proof of Theorem 3.8.

Lemma 4.1. Let $\mathcal{C}$ be an $\infty$-category admitting cotensors, and suppose we are given two space-valued functors $F, G \in \operatorname{Fun}(\mathcal{C}, \mathcal{S})$ that commute with cotensors. Then, a natural transformation $F \rightarrow G$ is a natural equivalence in $\mathrm{Fun}(\mathcal{C}, \mathcal{S})$ if and only if its postcomposition $\pi_{0} F \rightarrow \pi_{0} G$ with $\mathcal{S} \stackrel{\pi_{0}}{\longrightarrow}$ Set is a natural isomorphism in $\operatorname{Fun}(\mathcal{C}$, Set).

Proof. The "only if" direction is clear. So, suppose we are given a natural transformation $F \rightarrow G$ in Fun $(\mathcal{C}, \mathcal{S})$ such that the induced natural transformation $\pi_{0} F \rightarrow \pi_{0} G$ is a natural equivalence in Fun(e, Set). Since equivalences in $\operatorname{Fun}(\mathcal{C}, \mathcal{S})$ are determined componentwise, it suffices to show that for any $Y \in \mathcal{C}$, the map $F(Y) \rightarrow G(Y)$ is an equivalence in $\mathcal{S}$. In turn, since equivalences in $\mathcal{S}$ are created in ho(S), by Yoneda's lemma it suffices to show that for any $Z \in \mathcal{S}$, the induced map $[Z, F(Y)]_{\mathcal{S}} \rightarrow[Z, G(Y)]_{\mathcal{S}}$ is an isomorphism in $S$ et. But since $\mathcal{C}$ admits cotensors, then we can reidentify this map via the canonical commutative square

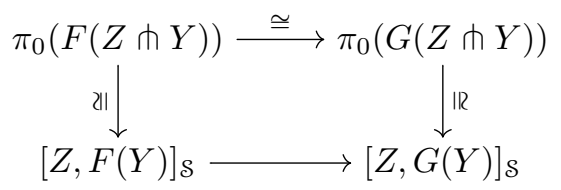

in Set, in which the top arrow is an isomorphism by the assumption that $\pi_{0} F \rightarrow \pi_{0} G$ is a natural isomorphism and the vertical arrows are isomorphisms by the assumption that $F$ and $G$ commute with cotensors. This proves the claim.

Before moving on to Lemma 4.3, it will be convenient to have the following bit of terminology.

Definition 4.2. A morphism in a model category $\mathcal{M}$ is called a homotopy epimorphism if it presents an epimorphism in the underlying $\infty$-category $\mathcal{M} \llbracket \mathbf{W}^{-1} \rrbracket$. 
We now proceed to the technical heart of the proof of Theorem 3.8. We warn the reader that our proof of the following result is (perhaps unexpectedly, and certainly unsatisfyingly) complicated.

Lemma 4.3. The map $\mathrm{N}_{\infty}(\mathcal{R}) \rightarrow \mathrm{L}_{\mathcal{e S S}}\left(\mathrm{N}_{\infty}^{\mathrm{R}}(\mathcal{R}, \mathbf{W})\right)$ is an epimorphism in CSS.

Proof. Our proof will proceed using model categories - primarily $s s \mathcal{S e t}_{\text {Rezk }}$ and $s \mathcal{S}_{\text {et }}$ Joyal $_{\text {, but also a number }}$ of others auxiliarily -, and will also use the language of marked simplicial sets (see e.g. §T.3.1).

We begin by recalling the two Quillen equivalences between $s s \mathcal{S e t}_{\text {Rezk }}$ and $s \mathcal{S e t}_{J o y a l}$ given in [JT07].

(1) Let us write $\boldsymbol{\Delta}^{o p} \times \boldsymbol{\Delta}^{o p} \stackrel{\mathrm{pr}_{2}}{\longrightarrow} \boldsymbol{\Delta}^{o p}$ for the second projection map and $\boldsymbol{\Delta}^{o p} \stackrel{i_{2}}{\longrightarrow} \boldsymbol{\Delta}^{o p} \times \boldsymbol{\Delta}^{o p}$ for the functor const $\left([0]^{\circ}\right) \times \operatorname{id}_{\Delta^{o p}}$. Pullbacks along these two functors induce the Quillen equivalence

$$
\mathrm{pr}_{2}^{*}: s \mathcal{S e t}_{\text {Joyal }} \rightleftarrows s s \mathcal{S e t}_{\text {Rezk }}: i_{2}^{*}
$$

of [JT07, Theorem 4.11].

(2) Let us write $\left(\Delta^{i}\right)^{\text {gpd }} \in s \mathcal{S}$ et for the nerve of the strict (i.e. objects-preserving) groupoid completion of $[i] \in$ eat, and let us write $t_{!}: s s \mathcal{S e t} \rightarrow s \mathcal{S}$ et for the left Kan extension

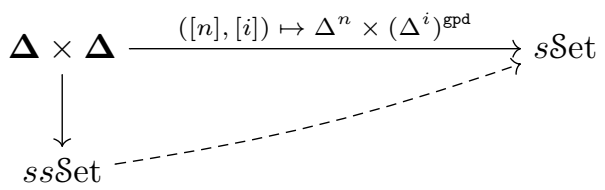

along the (1-categorical) Yoneda embedding. This has a right adjoint $t^{!}: s \mathcal{S}$ et $\rightarrow s s \mathcal{S e t}$ given by

$$
t^{!}(Y)=\left\{\left\{\operatorname{hom}_{s \text { et }}\left(\Delta^{n} \times\left(\Delta^{i}\right)^{\text {gpd }}, Y\right)\right\}_{i \geq 0}\right\}_{n \geq 0},
$$

and together these fit into the Quillen equivalence

$$
t_{!}: s s \mathcal{S e t}_{\text {Rezk }} \rightleftarrows s \mathcal{S e t}_{\text {Joyal }}: t^{!}
$$

of [JT07, Theorem 4.12].

Now, suppose that $\mathrm{R} \in s \mathcal{S e t}_{\text {Joyal }}^{f}$ is a quasicategory presenting $\mathcal{R} \in \mathcal{C a t}_{\infty}$, and let $(\mathrm{R}, \mathrm{W}) \in s \mathcal{S e t}^{+}$be the marked simplicial set obtained by marking precisely those edges of $\mathrm{R}$ which present maps in $\mathbf{W} \subset \mathcal{R}$. For any $n \geq 0$, the $\infty$-category $\operatorname{Fun}([n], \mathcal{R})$ is presented by the object

$$
\underline{\operatorname{hom}}_{s \text { Set }}\left(\Delta^{n}, \mathrm{R}\right)=\left\{\operatorname{hom}_{s \text { Set }}\left(\Delta^{n} \times \Delta^{i}, \mathrm{R}\right)\right\}_{i \geq 0} \in s \operatorname{set}_{\text {Joyal }},
$$

and hence its subcategory

$$
\operatorname{Fun}([n], \mathcal{R})^{\mathbf{W}} \subset \operatorname{Fun}([n], \mathcal{R})
$$

is presented by the object

$$
\left\{\operatorname{hom}_{\text {sset }^{+}}\left(\left(\Delta^{n}\right)^{\mathrm{b}} \times\left(\Delta^{i}\right)^{\sharp},(\mathrm{R}, \mathrm{W})\right)\right\}_{i \geq 0} \in \text { s.et Joyal }_{\text {J }} .
$$

These constructions are contravariantly functorial in $[n] \in \boldsymbol{\Delta}$, and hence we obtain that the Rezk pre-nerve

$$
\operatorname{preN}_{\infty}^{\mathrm{R}}(\mathcal{R}, \mathbf{W})=\operatorname{Fun}^{\mathrm{lw}}([\bullet], \mathcal{R})^{\mathbf{W}} \in \operatorname{seat}_{\infty}
$$

is presented by the object

$$
\left\{\left\{\text { hom }_{\text {sset }}+\left(\left(\Delta^{n}\right)^{\mathrm{b}} \times\left(\Delta^{i}\right)^{\sharp},(\mathrm{R}, \mathrm{W})\right)\right\}_{i \geq 0}\right\}_{n \geq 0} \in s\left(\text { SSet Joyal }_{\text {Reedy }} .\right.
$$

From here, we observe that the Quillen adjunction

$$
\operatorname{id}_{s s S e t}: s\left(s \mathcal{S e t}_{\text {Joyal }}\right)_{\text {Reedy }} \rightleftarrows s\left(s \mathcal{S e t}_{\mathrm{KQ}}\right)_{\text {Reedy }}: \operatorname{id}_{s s \mathcal{S e t}}
$$

presents the left localization adjunction $s\left((-)^{\text {gpd }}\right): s \mathcal{C a t}_{\infty} \rightleftarrows s \mathcal{S}: s\left(\mathrm{U}_{\mathcal{S}}\right)$; as all objects of $s(s \mathcal{S} \text { et Joyal })_{\text {Reedy }}$ are cofibrant, it follows that when considered as an object of $s\left(s \mathcal{S} \text { et }{ }_{\mathrm{KQ}}\right)_{\text {Reedy }}$, this same bisimplicial set presents $\mathrm{N}_{\infty}^{\mathrm{R}}(\mathcal{R}, \mathbf{W}) \in s \mathcal{S}$. Moreover, in light of the left Bousfield localization

$$
\operatorname{id}_{s s S e t}: s\left(s \mathcal{S e t}_{\mathrm{KQ}}\right)_{\text {Reedy }} \rightleftarrows s s \mathcal{S e t} \operatorname{Rezk}: \mathrm{id}_{s s S e t}
$$


presenting the left localization adjunction $\mathrm{L}_{\mathcal{C S S}}: s \mathcal{S} \rightleftarrows \mathcal{C S S}: \mathrm{U}_{\mathcal{E S S}}$, when considered as an object of $s s \mathcal{S}$ et $\mathrm{Rezk}_{\text {, }}$ this same bisimplicial set presents the Rezk nerve

$$
\mathrm{N}_{\infty}^{\mathrm{R}}(\mathcal{R}, \mathbf{W})=\left(\operatorname{Fun}^{\mathrm{lw}}([\bullet], \mathcal{R})^{\mathbf{W}}\right)^{\text {gpd }} \in \mathcal{E S S} .
$$

We will denote this bisimplicial set by $\mathrm{N}^{\mathrm{R}}(\mathrm{R}, \mathrm{W}) \in \operatorname{ssSet}^{10}$ In particular, note that we have a natural isomorphism $\mathrm{N}^{\mathrm{R}}\left(\mathrm{R}^{\natural}\right) \cong t^{!}(\mathrm{R})$ in $s s \mathcal{S}$ et, and hence we see that the right Quillen equivalence

$$
t^{!}: s \mathcal{S e t}_{\text {Joyal }} \rightarrow s s \text { Set }_{\text {Rezk }}
$$

presents the equivalence $\mathrm{N}_{\infty}$ : Cat $_{\infty} \stackrel{\sim}{\longrightarrow}$ eSS of $\infty$-categories.

Now, the natural map

$$
\mathrm{R}^{\natural} \rightarrow(\mathrm{R}, \mathrm{W})
$$

in $s$ Set $^{+}$induces a map

$$
\mathrm{N}^{\mathrm{R}}\left(\mathrm{R}^{\natural}\right) \rightarrow \mathrm{N}^{\mathrm{R}}(\mathrm{R}, \mathrm{W})
$$

in $s s \mathcal{S e t}_{\text {Rezk }}$, which by what we have seen presents the map

$$
\mathrm{N}_{\infty}(\mathcal{R}) \rightarrow \operatorname{Less}_{\mathcal{e s}}\left(\mathrm{N}_{\infty}^{\mathrm{R}}(\mathcal{R}, \mathbf{W})\right)
$$

in $\mathcal{C S S}$. So, to prove that this latter map is an epimorphism in $\mathcal{C S S}$, it suffices to prove that the former map is a homotopy epimorphism in $s s \mathcal{S e t}_{\text {Rezk }}$. However, note that there is a natural isomorphism $t_{!}\left(\operatorname{pr}_{2}^{*}(\mathrm{R})\right) \cong \mathrm{R}$ in $s \mathcal{S e t}$, which is in particular a weak equivalence in $s \mathcal{S e t}_{\text {Joyal }}$; via the Quillen equivalence of item (2), this corresponds to a weak equivalence $\mathrm{pr}_{2}^{*}(\mathrm{R}) \stackrel{\approx}{\rightarrow} t^{!}(\mathrm{R})$ in $s s \mathcal{S e t}_{\text {Rezk }}$. So, it also suffices to show that the composite map

$$
\operatorname{pr}_{2}^{*}(\mathrm{R}) \stackrel{\approx}{\rightarrow} t^{!}(\mathrm{R}) \cong \mathrm{N}^{\mathrm{R}}\left(\mathrm{R}^{\natural}\right) \rightarrow \mathrm{N}^{\mathrm{R}}(\mathrm{R}, \mathrm{W})
$$

is a homotopy epimorphism in $s s \mathcal{S e t}_{\text {Rezk }}$.

For this, let us also recall the "usual" geometric realization functor $s s \mathcal{S e t} \rightarrow s \mathcal{S e t}$ (a homotopy colimit functor with respect to $\left.s\left(s \mathcal{S e t}_{\mathrm{KQ}}\right)_{\text {Reedy }}\right)$ : this is the left Kan extension

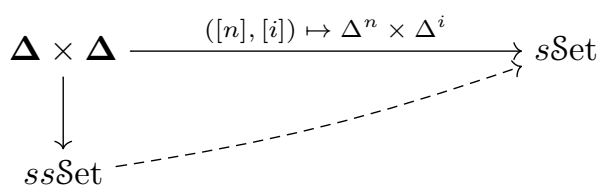

along the (1-categorical) Yoneda embedding, but by [GJ99, Chapter IV, Exercise 1.6] this is (naturally isomorphic to) the functor $\operatorname{diag}^{*}: s s \mathcal{S e t} \rightarrow s \mathcal{S e t}$, where $\boldsymbol{\Delta}^{o p} \stackrel{\text { diag }}{\longrightarrow} \boldsymbol{\Delta}^{o p} \times \boldsymbol{\Delta}^{o p}$ denotes the diagonal functor. Now, the evident morphisms $\Delta^{n} \times \Delta^{i} \rightarrow \Delta^{n} \times\left(\Delta^{i}\right)^{\text {gpd }}$ in $s$ Set induce a natural transformation diag* $\rightarrow t_{\text {! }}$ in Fun(ssSet, $s \mathcal{S e t})$. Moreover, it is not hard to see that upon precomposition with $s \mathcal{S e t} \stackrel{\mathrm{pr}_{2}^{*}}{\longrightarrow} s s \mathcal{S e t}$, this induces the identity natural transformation from $\operatorname{id}_{s \mathcal{S} \text { et }}$ to itself in Fun( $s \mathcal{S}$ et, $s \mathcal{S}$ et) (up to isomorphism). Applying these observations to the above composite map in $s s \mathcal{S}$ et, we obtain a commutative square

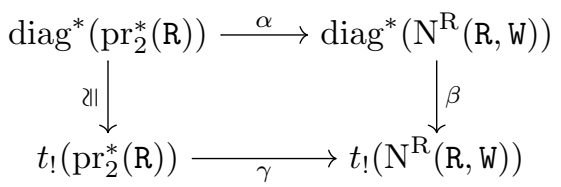

in $s \mathcal{S e t}$, where both objects on the left are (compatibly) isomorphic to R itself. Since $t_{!}: s s \mathcal{S e t}_{\text {Rezk }} \rightarrow s \mathcal{S}$ et ${ }_{J o y a l}$ is a left Quillen equivalence and all objects of $s s \mathcal{S e t}_{\text {Rezk }}$ are cofibrant, it suffices to show that the map $\gamma$ is a homotopy epimorphism in $s \mathcal{S e t}_{\text {Joyal }}$. For this, it suffices to prove that when considered in $s \mathcal{S e t}$ Joyal $_{\text {, the map }}$ $\alpha$ is a weak equivalence and the map $\beta$ is a homotopy epimorphism. This, finally, is what we will show.

We begin with the second assertion, that the map

$$
\operatorname{diag}^{*}\left(\mathrm{~N}^{\mathrm{R}}(\mathrm{R}, \mathrm{W})\right) \stackrel{\beta}{\rightarrow} t_{!}\left(\mathrm{N}^{\mathrm{R}}(\mathrm{R}, \mathrm{W})\right)
$$

\footnotetext{
${ }^{10}$ When $(\mathrm{R}, \mathrm{W}) \in s \operatorname{Set}^{+}$is the "marked nerve" of a relative 1-category, this recovers the 1-categorical Rezk nerve of Remark 3.2 (as an object of $s s S e t$ ), and so there is no ambiguity in the notation.
} 
is a homotopy epimorphism in $s \mathcal{S e t}_{\text {Joyal }}$. In fact, we will show that the natural transformation $\operatorname{diag}^{*} \rightarrow t_{\text {! }}$ in Fun(sset, $s \mathcal{S}_{\text {et }}$ Joyal $)$ is a componentwise homotopy epimorphism. Just for the duration of this sub-proof, let us "reverse" our simplicial coordinates, so that the one we have been denoting by " $"$ " will be the outer coordinate while the one we have been denoting by " $n$ " will be the inner coordinate. Now, observe that we can rewrite these two functors as

$$
\operatorname{diag}^{*} \cong \int^{[i] \in \Delta}(-)_{i} \times \Delta^{i}: s(s \mathcal{S e t}) \rightarrow s \mathcal{S e t}
$$

and

$$
t_{!} \cong \int^{[i] \in \Delta}(-)_{i} \times\left(\Delta^{i}\right)^{\text {gpd }}: s(s \mathcal{S e t}) \rightarrow s \mathcal{S e t},
$$

under which identifications our natural transformation diag* $\rightarrow t_{\text {! }}$ is induced by the evident map $\Delta^{\bullet} \rightarrow$ $\left(\Delta^{\bullet}\right)^{\text {gpd }}$ in $c(s \mathcal{S e t})$. Moreover, by Proposition T.A.2.9.26, we obtain a left Quillen bifunctor

$$
\int^{[i] \in \boldsymbol{\Delta}}(-)_{i} \times(-)^{i}: s\left(s \operatorname{Set}_{\text {Joyal }}\right)_{\text {Reedy }} \times c\left(s \operatorname{Set}_{\text {Joyal }}\right)_{\text {Reedy }} \rightarrow s \operatorname{Set}_{\text {Joyal }}
$$

(since $s$ Set $_{\text {Joyal }}$ is cartesian, i.e. the product bifunctor is left Quillen). ${ }^{11}$ As every object of $s$ (sSet Joyal) Reedy is cofibrant, for any object

$$
Y_{\bullet} \in s\left(\text { sSet Joyal }_{\text {Reedy }}\right.
$$

the above left Quillen bifunctor induces a left Quillen functor

$$
\int^{[i] \in \Delta} Y_{i} \times(-)^{i}: c\left(s \mathcal{S e t}_{\text {Joyal }}\right)_{\text {Reedy }} \rightarrow s \operatorname{Set}_{\text {Joyal }} \text {. }
$$

Moreover, the cofibrant objects of $c(s \mathcal{S} \text { et Joyal })_{\text {Reedy }}$ are exactly those of $c\left(s \mathcal{S e t}_{\mathrm{KQ}}\right)_{\text {Reedy }}$ ( since the cofibrations in $s \mathcal{S e t}_{\text {Joyal }}$ are exactly those of $\left.s \mathcal{S e t}_{\mathrm{KQ}}\right)$, and so in particular the objects $\Delta^{\bullet},\left(\Delta^{\bullet}\right)^{\mathrm{gpd}} \in c\left(\operatorname{sSet}_{\text {Joyal }}\right)_{\text {Reedy }}$ are cofibrant by [Hir03, Corollary 15.9.10].

Now, epimorphisms (being determined by a colimit condition) are preserved by left adjoint functors of $\infty$-categories. Moreover, by [MGq, Theorem 2.1], a left Quillen functor between model categories induces a left adjoint functor between $\infty$-categories, which is presented (in Releat $_{\mathrm{BK}}$ ) by the restriction of the left Quillen functor to the subcategory of cofibrant objects. So, it suffices to show that the map $\Delta^{\bullet} \rightarrow\left(\Delta^{\bullet}\right)^{\text {gpd }}$ is a homotopy epimorphism in $c\left(s \text { Set }_{\text {Joyal }}\right)_{\text {Reedy }}$.

For this, observe that the model category $c\left(s \mathcal{S e t}_{\text {Joyal }}\right)_{\text {Reedy }}$ presents the $\infty$-category $c \mathcal{C}_{\infty}$ t $_{\infty}$. Since epimorphisms in $c$ Cat $_{\infty}=\operatorname{Fun}\left(\boldsymbol{\Delta}\right.$, Cat $\left._{\infty}\right)$ are determined componentwise, it suffices to show that each $\Delta^{i} \rightarrow\left(\Delta^{i}\right)^{\mathrm{gpd}}$ is a homotopy epimorphism in $s \mathcal{S}_{\text {et Joyal }}$. But this is clear: this map in $s \mathcal{S e t}_{\text {Joyal }}$ presents the terminal map

$$
[i] \rightarrow[i]^{\text {gpd }} \simeq \operatorname{pt}_{e_{\text {at }}}
$$

in $\mathcal{C a t}_{\infty}$, which on an arbitrary $\infty$-category $\mathcal{C}$ corepresents the inclusion

$$
\mathrm{e} \simeq \hookrightarrow \operatorname{hom}_{\mathrm{Cat}_{\infty}}([i], \mathcal{C})
$$

of the subspace of length- $i$ sequences of composable equivalences (inside of the space of arbitrary length- $i$ sequences of composable morphisms). Thus, the natural transformation $\operatorname{diag}^{*} \rightarrow t_{\text {! }}$ in Fun(ssSet, $s$ Set Joyal $)$ is indeed a componentwise homotopy epimorphism, and so in particular we obtain that the map $\beta$ (which is its component at the object $\left.\mathrm{N}^{\mathrm{R}}(\mathrm{R}, \mathrm{W}) \in s s \mathrm{Set}\right)$ is a homotopy epimorphism, as claimed.

So, it only remains to show that the map

$$
\mathrm{R} \cong \operatorname{diag}^{*}\left(\operatorname{pr}_{2}^{*}(\mathrm{R})\right) \stackrel{\alpha}{\rightarrow} \operatorname{diag}^{*}\left(\mathrm{~N}^{\mathrm{R}}(\mathrm{R}, \mathrm{W})\right)
$$

is a weak equivalence in $s \mathcal{S}_{\text {et }}$ Joyal. Unwinding the definitions, we see that via the evident cosimplicial object

$$
\Delta \stackrel{\left(\Delta^{\bullet}\right)^{b} \times\left(\Delta^{\bullet}\right)^{\sharp}}{\longrightarrow} s \operatorname{Set}^{+},
$$

we obtain a canonical isomorphism

$$
\operatorname{diag}^{*}\left(\mathrm{~N}^{\mathrm{R}}(\mathrm{R}, \mathrm{W})\right) \cong \operatorname{hom}_{s \operatorname{set}^{+}}^{\mathrm{lw}}\left(\left(\Delta^{\bullet}\right)^{\mathrm{b}} \times\left(\Delta^{\bullet}\right)^{\sharp},(\mathrm{R}, \mathrm{W})\right) .
$$

\footnotetext{
${ }^{11}$ Note that since we have flipped our simplicial coordinates, this model structure $s\left(s \mathcal{S e t}\right.$ Joyal $_{\text {Reedy }}$ is different from the model structure $s\left(s \mathcal{S}\right.$ J Joyal $_{\text {Reedy }}$ that appeared earlier (with respect to the fixed copy of the underlying category $s s \mathcal{S e t}$ in which we have been working).
} 
Moreover, via the canonical isomorphisms

$$
\mathrm{R} \cong \operatorname{hom}_{s \text { Set }}^{\mathrm{lw}}\left(\Delta^{\bullet}, \mathrm{R}\right) \cong \operatorname{hom}_{s \operatorname{Set}^{+}}^{\mathrm{lw}}\left(\left(\Delta^{\bullet}\right)^{\mathrm{b}}, \mathrm{R}^{\mathrm{b}}\right) \cong \operatorname{hom}_{s \operatorname{Set}^{+}}^{\mathrm{lw}}\left(\left(\Delta^{\bullet}\right)^{\mathrm{b}},(\mathrm{R}, \mathrm{W})\right),
$$

this map $\alpha$ is corepresented by the collection of first projection maps

$$
\left(\Delta^{n}\right)^{b} \times\left(\Delta^{n}\right)^{\sharp} \rightarrow\left(\Delta^{n}\right)^{b},
$$

which assemble to a natural transformation in $\operatorname{Fun}\left(\boldsymbol{\Delta}, s \operatorname{Set}^{+}\right)$. On the other hand, the collection of diagonal maps

$$
\left(\Delta^{n}\right)^{b} \rightarrow\left(\Delta^{n}\right)^{b} \times\left(\Delta^{n}\right)^{\sharp}
$$

(or more precisely, the unique maps in $s \mathcal{S e t}^{+}$which recover the diagonal maps in sSet under the forgetful functor $\left.s \mathcal{S e t}^{+} \rightarrow s \mathcal{S e t}\right)$ also assemble into a natural transformation in $\operatorname{Fun}\left(\boldsymbol{\Delta}, s \mathcal{S e t}^{+}\right)$, which likewise corepresents a map

in $s$ Set. Clearly, the composite

$$
\operatorname{diag}^{*}\left(\mathrm{~N}^{\mathrm{R}}(\mathrm{R}, \mathrm{W})\right) \stackrel{\rho}{\rightarrow} \mathrm{R}
$$

$$
\mathrm{R} \stackrel{\alpha}{\rightarrow} \operatorname{diag}^{*}\left(\mathrm{~N}^{\mathrm{R}}(\mathrm{R}, \mathrm{W})\right) \stackrel{\rho}{\rightarrow} \mathrm{R}
$$

is the identity map, since this is true of the composite

$$
\left(\Delta^{n}\right)^{b} \rightarrow\left(\Delta^{n}\right)^{b} \times\left(\Delta^{n}\right)^{\sharp} \rightarrow\left(\Delta^{n}\right)^{b}
$$

of the diagonal map followed by the first projection. On the other hand, we will show that the composite

$$
\operatorname{diag}^{*}\left(\mathrm{~N}^{\mathrm{R}}(\mathrm{R}, \mathrm{W})\right) \stackrel{\rho}{\rightarrow} \mathrm{R} \stackrel{\alpha}{\rightarrow} \operatorname{diag}^{*}\left(\mathrm{~N}^{\mathrm{R}}(\mathrm{R}, \mathrm{W})\right)
$$

is connected to $\operatorname{id}_{\operatorname{diag}^{*}\left(N^{R}(R, W)\right)}$ by the zigzag of simplicial homotopies illustrated in Figure 1, whose compo-

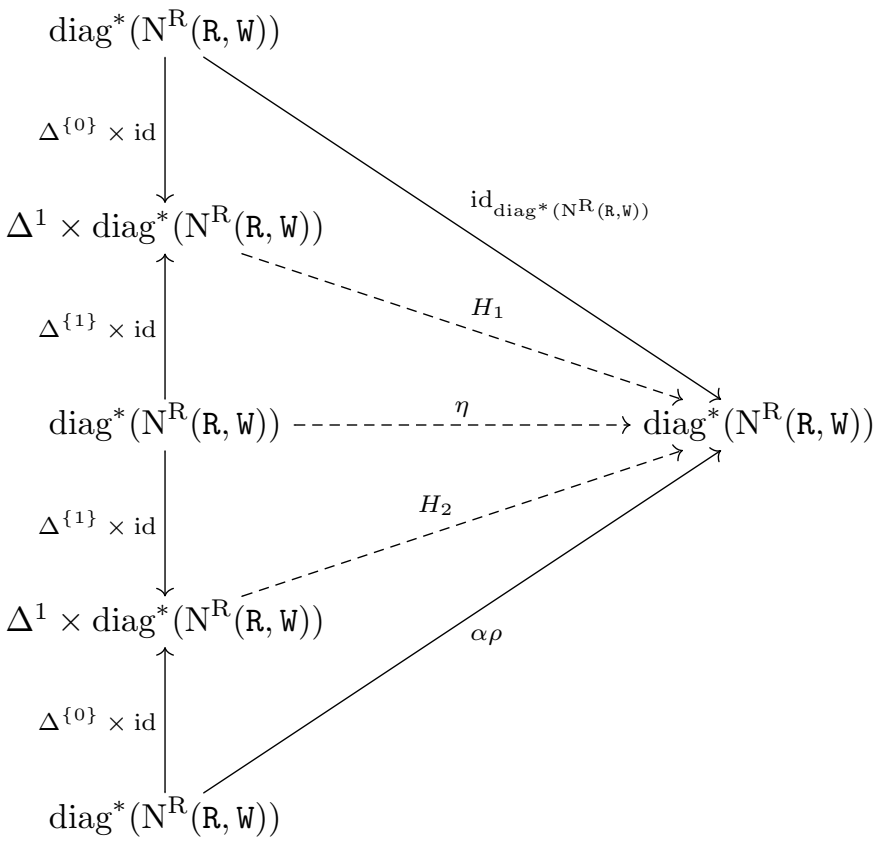

Figure 1. The zigzag of simplicial homotopies in $s$ Set in the proof of Lemma 4.3 .

nents (i.e. whose values on the vertices of (the source copies of $\left.) \operatorname{diag}^{*}\left(N^{R}(R, W)\right)\right)$ are all degenerate edges of (the target copy of) $\operatorname{diag}^{*}\left(\mathrm{~N}^{\mathrm{R}}(\mathrm{R}, \mathrm{W})\right)$. Postcomposing with an arbitrary fibrant replacement

$$
\operatorname{diag}^{*}\left(\mathrm{~N}^{\mathrm{R}}(\mathrm{R}, \mathrm{W})\right) \stackrel{\widetilde{\rightarrow}}{\mathbb{R}}\left(\operatorname{diag}^{*}\left(\mathrm{~N}^{\mathrm{R}}(\mathrm{R}, \mathrm{W})\right)\right) \rightarrow \mathrm{pt}_{\text {sset }}
$$

in $s \mathcal{S e t}_{\text {Joyal }}$, we obtain a composite

$$
\Lambda_{2}^{2} \rightarrow \underline{\operatorname{hom}}_{s \operatorname{set}}\left(\operatorname{diag}^{*}\left(\mathrm{~N}^{\mathrm{R}}(\mathrm{R}, \mathrm{W})\right), \operatorname{diag}^{*}\left(\mathrm{~N}^{\mathrm{R}}(\mathrm{R}, \mathrm{W})\right)\right) \rightarrow \underline{\operatorname{hom}}_{s \operatorname{set}}\left(\operatorname{diag}^{*}\left(\mathrm{~N}^{\mathrm{R}}(\mathrm{R}, \mathrm{W})\right), \mathbb{R}\left(\operatorname{diag}^{*}\left(\mathrm{~N}^{\mathrm{R}}(\mathrm{R}, \mathrm{W})\right)\right)\right)
$$




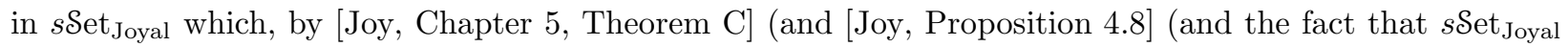

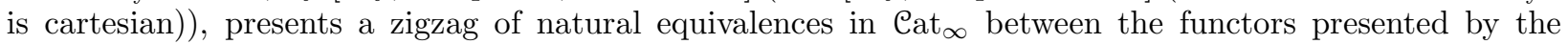
maps $\operatorname{id}_{\operatorname{diag}^{*}\left(\mathrm{~N}^{\mathrm{R}}(\mathrm{R}, \mathrm{W})\right)}$ and $\alpha \rho$ in $s \mathcal{S e t}_{\text {Joyal }}$. In turn, this zigzag (along with the natural equivalence in $\operatorname{Cat}_{\infty}$ presented by the identification $\rho \alpha=\mathrm{id}_{\mathrm{R}}$ ) witnesses the fact that the maps $\alpha$ and $\rho$ in $s \mathcal{S}_{\text {et Joyal }}$ present inverse equivalences in $\mathrm{Cat}_{\infty}$, from which we conclude that in particular the map $\alpha$ is indeed a weak equivalence in $s$ Set $_{\text {Joyal }}$.

Now, all three of $\eta, H_{1}$, and $H_{2}$ will be corepresented by maps between the various objects $\left(\Delta^{n}\right)^{b} \times$ $\left(\Delta^{n}\right)^{\sharp} \in s$ Set $^{+}$; in turn, all of these maps will be obtained by applying the evident "marked nerve" functor $\mathrm{N}^{+}$: Releat $\rightarrow s$ Set $^{+}$to maps between the various objects $[n] \times[n]_{\mathrm{W}} \in$ Releat.

We begin by defining the map $\operatorname{diag}^{*}\left(\mathrm{~N}^{\mathrm{R}}(\mathrm{R}, \mathrm{W})\right) \stackrel{\eta}{\rightarrow} \operatorname{diag}^{*}\left(\mathrm{~N}^{\mathrm{R}}(\mathrm{R}, \mathrm{W})\right)$ : this is corepresented by the marked nerves of the maps

$$
[n] \times[n]_{\mathbf{W}} \stackrel{\eta^{n}}{\longrightarrow}[n] \times[n]_{\mathbf{W}}
$$

in Releat given by

$$
\eta^{n}(i, j)= \begin{cases}(i, i), & i \geq j \\ (i, j), & i<j\end{cases}
$$

It is easy to verify that this does indeed define a map in releat, and moreover that assembling these maps for all $n \geq 0$ yields an endomorphism of the object $[\bullet] \times[\bullet]_{\mathrm{W}} \in$ creleat.

In order to define the simplicial homotopies $H_{1}$ and $H_{2}$, we first recall a combinatorial reformation of the definition of a simplicial homotopy (see e.g. [May92, Definitions 5.1]): for any $Y, Z \in s \mathcal{S e t}$ and any $f, g \in \operatorname{hom}_{s \operatorname{set}}(Y, Z)$, a simplicial homotopy

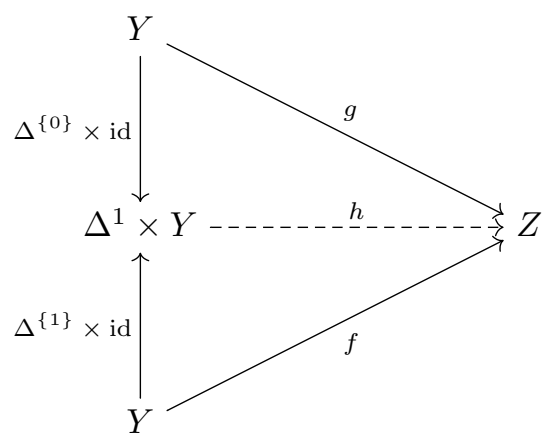

is equivalently given by a family of maps

$$
\left\{h_{i, n} \in \operatorname{hom}_{\mathcal{S e t}}\left(Y_{n}, Z_{n+1}\right)\right\}_{0 \leq i \leq n \geq 0}
$$

which satisfy the identities

$$
\begin{gathered}
\delta_{0} h_{0, n}=f_{n}, \\
\delta_{n+1} h_{n, n}=g_{n}, \\
\delta_{i} h_{j, n}= \begin{cases}h_{j-1, n-1} \delta_{i}, & i<j \\
\delta_{i} h_{i-1, n}, & i=j \neq 0 \\
h_{j, n-1} \delta_{i-1}, & i>j+1,\end{cases}
\end{gathered}
$$

and

$$
\sigma_{i} h_{j, n}= \begin{cases}h_{j+1, n+1} \sigma_{i}, & i \leq j \\ h_{j, n+1} \sigma_{i-1}, & i>j\end{cases}
$$

So, for $\varepsilon \in\{1,2\}$, we will define the simplicial homotopies

$$
\Delta^{1} \times \operatorname{diag}^{*}\left(\mathrm{~N}^{\mathrm{R}}(\mathrm{R}, \mathrm{W})\right) \stackrel{H_{\varepsilon}}{\longrightarrow} \operatorname{diag}^{*}\left(\mathrm{~N}^{\mathrm{R}}(\mathrm{R}, \mathrm{W})\right)
$$

to be corepresented by the marked nerves of families of maps

$$
\left\{H_{\varepsilon}^{i, n} \in \operatorname{hom}_{\text {releat }}\left([n+1] \times[n+1]_{\mathbf{W}},[n] \times[n]_{\mathbf{W}}\right)\right\}_{0 \leq i \leq n \geq 0}
$$


satisfying the opposites of the identities given above (with the first two "boundary condition" identities being dictated by their respective sources and targets). Namely, we define

$$
H_{1}^{i, n}(j, k)= \begin{cases}(j, k), & 0 \leq j, k \leq i \\ (j-1, j-1), & j>i \text { and } j \geq k \\ (j, k-1), & k>i \geq j \\ (j-1, k-1), & k>j>i\end{cases}
$$

and

$$
H_{2}^{i, n}(j, k)= \begin{cases}(j, j), & j \leq i \\ (j-1, j-1), & j>i \text { and } j \geq k \\ (j-1, k-1), & k>j>i .\end{cases}
$$

It is a straightforward (but lengthy) process to verify

- that these satisfy the opposites of the identities given above,

- that they restrict along their boundaries to the various maps

$$
\operatorname{id}_{\text {diag* }\left(\mathrm{N}^{\mathrm{R}}(\mathrm{R}, \mathrm{W})\right)}, \eta, \alpha \rho \in \operatorname{hom}_{s \operatorname{set}}\left(\operatorname{diag}^{*}\left(\mathrm{~N}^{\mathrm{R}}(\mathrm{R}, \mathrm{W})\right), \operatorname{diag}^{*}\left(\mathrm{~N}^{\mathrm{R}}(\mathrm{R}, \mathrm{W})\right)\right)
$$

as indicated in Figure 1, and

- that their values on vertices are all degenerate edges,

as claimed. This completes the proof.

\section{REFERENCES}

[BK12] C. Barwick and D. M. Kan, Relative categories: another model for the homotopy theory of homotopy theories, Indag. Math. (N.S.) 23 (2012), no. 1-2, 42-68.

[GJ99] Paul G. Goerss and John F. Jardine, Simplicial homotopy theory, Progress in Mathematics, vol. 174, Birkhäuser Verlag, Basel, 1999.

[Hir03] Philip S. Hirschhorn, Model categories and their localizations, Mathematical Surveys and Monographs, vol. 99, American Mathematical Society, Providence, RI, 2003.

[Joy] André Joyal, The Theory of Quasi-Categories and its Applications, Volume II, available at http://mat.uab.cat/ kock/crm/hocat/advanced-course/Quadern45-2.pdf.

[JT07] André Joyal and Myles Tierney, Quasi-categories vs Segal spaces, Categories in algebra, geometry and mathematical physics, Contemp. Math., vol. 431, Amer. Math. Soc., Providence, RI, 2007, pp. 277-326.

[LMG15] Zhen Lin Low and Aaron Mazel-Gee, From fractions to complete Segal spaces, Homology Homotopy Appl. 17 (2015), no. $1,321-338$.

[Lur09a] Jacob Lurie, Higher topos theory, Annals of Mathematics Studies, vol. 170, Princeton University Press, Princeton, NJ, 2009, also available at http://math.harvard.edu/ lurie and at arXiv:math/0608040, v4.

[Lur09b] _ , $(\infty, 2)$-categories and the Goodwillie calculus $I$, available at http://www.math.harvard.edu/ lurie, version dated October 8, 2009.

[Lur14] , Higher algebra, available at http://www.math.harvard.edu/ lurie, version dated September 14, 2014.

[May92] J. Peter May, Simplicial objects in algebraic topology, Chicago Lectures in Mathematics, University of Chicago Press, Chicago, IL, 1992, Reprint of the 1967 original.

[MGa] Aaron Mazel-Gee, Model $\infty$-categories I: some pleasant properties of the $\infty$-category of simplicial spaces, available at arXiv:1412.8411, v2.

[MGc] , All about the Grothendieck construction, to appear.

[MGd] Hammocks and fractions in relative $\infty$-categories, to appear.

[MGe] - Model $\infty$-categories II: Quillen adjunctions, to appear.

[MGf] Model $\infty$-categories III: the fundamental theorem, to appear.

[MGq] Quillen adjunctions induce adjunctions of quasicategories, available at arXiv:1501.03146, v1.

[Rez01] Charles Rezk, A model for the homotopy theory of homotopy theory, Trans. Amer. Math. Soc. 353 (2001), no. 3, 973-1007 (electronic).

[Toë05] Bertrand Toën, Vers une axiomatisation de la théorie des catégories supérieures, K-Theory 34 (2005), no. 3, $233-263$. 